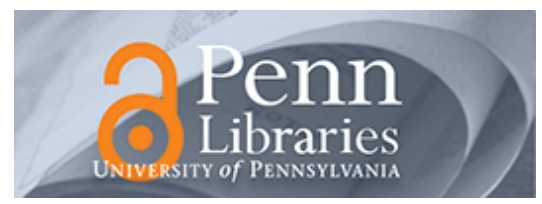

University of Pennsylvania

ScholarlyCommons

August 1982

\title{
The Stability of Second Order Quadratic Differential Equations
}

\author{
Daniel E. Koditschek \\ University of Pennsylvania, kod@seas.upenn.edu \\ Kumpati J. Narendra \\ Yale University
}

Follow this and additional works at: https://repository.upenn.edu/ese_papers

\section{Recommended Citation}

Daniel E. Koditschek and Kumpati J. Narendra, "The Stability of Second Order Quadratic Differential Equations", . August 1982.

Copyright YEAR 1982. Reprinted from IEEE Transactions on Automatic Control, Volume AC-27, Issue 4, August 1982, pages 783-798.

This material is posted here with permission of the IEEE. Such permission of the IEEE does not in any way imply IEEE endorsement of any of the University of Pennsylvania's products or services. Internal or personal use of this material is permitted. However, permission to reprint/republish this material for advertising or promotional purposes or for creating new collective works for resale or redistribution must be obtained from the IEEE by writing to pubs-permissions@ieee.org. By choosing to view this document, you agree to all provisions of the copyright laws protecting it.

At the time of publication, the author, Daniel Koditschek, was affiliated with Yale University. Currently, he is a member of the faculty of the School of Engineering at the University of Pennsylvania.

This paper is posted at ScholarlyCommons. https://repository.upenn.edu/ese_papers/342

For more information, please contact repository@pobox.upenn.edu. 


\title{
The Stability of Second Order Quadratic Differential Equations
}

\begin{abstract}
This paper investigates the stability properties of second-order systems, $x=f(x)$, where $f(x)$ contains either quadratic terms-system (1)-or linear and quadratic terms-system (2)-in $x$. The principal contributions are summarized in two theorems which give necessary and sufficient conditions for stability and asymptotic stability in the large of systems (1) and (2), respectively.

\section{Comments}

Copyright YEAR 1982. Reprinted from IEEE Transactions on Automatic Control, Volume AC-27, Issue 4, August 1982, pages 783-798.

This material is posted here with permission of the IEEE. Such permission of the IEEE does not in any way imply IEEE endorsement of any of the University of Pennsylvania's products or services. Internal or personal use of this material is permitted. However, permission to reprint/republish this material for advertising or promotional purposes or for creating new collective works for resale or redistribution must be obtained from the IEEE by writing to pubs-permissions@ieee.org. By choosing to view this document, you agree to all provisions of the copyright laws protecting it.

At the time of publication, the author, Daniel Koditschek, was affiliated with Yale University. Currently, he is a member of the faculty of the School of Engineering at the University of Pennsylvania.
\end{abstract}




\title{
The Stability of Second-Order Quadratic Differential Equations
}

\author{
DANIEL E. KODITSCHEK AND KUMPATI S. NARENDRA, FELLOW, IEEE
}

\begin{abstract}
This paper investigates the stability properties of second-order systems, $\dot{x}=f(x)$, where $f(x)$ contains either quadratic terms-system (1)-or linear and quadratic terms-system (2)-in $x$. The principal contributions are summarized in two theorems which give necessary and sufficient conditions for stability and asymptotic stability in the large of systems (1) and (2), respectively.
\end{abstract}

\section{INTRODUCTION}

$\mathbf{T}$ HE DIVERSE behavior of nonlinear systems reflects the obvious fact that no one theory can address all phenomena of interest in complex situations. Powerful techniques have evolved over the years in the context of specific questions regarding particular classes of such systems. This paper suggests a useful approach to the investigation of the stability characteristics of a class of second-order differential equations

$$
\dot{x} \triangleq\left[\begin{array}{c}
\dot{x}_{1} \\
\dot{x}_{2}
\end{array}\right]=\left[\begin{array}{c}
x^{T} G x \\
x^{T} H x
\end{array}\right] \triangleq B(x)
$$

and

$$
\dot{x}=A x+B(x) \triangleq f(x) .
$$

More than a convenient arbitrary choice, quadratic differential equations have a traditional place in the general literature, and an increasing importance in the field of systems theory. Historically, there has been a long standing interest on the part of pure and applied mathematicians in the behavior of planar quadratic systems. There have been numerous attempts to characterize the number and location of limit cycles of such systems, e.g., [11], [13]-[15]. In the last two decades, several exhaustive accounts of the integral curves of a pure quadratic system (1) have been presented [2], [6], [9], [10], [12] using a variety of algebraic or analytical tools. Coppel [1] has given a short, but useful survey of the historical literature. Differential equations of this type are known to arise in adaptive control where control parameters become state variables of a quadratic system. Recently, the special class of bilinear systems

$$
\dot{x}=A x+u D x+b u
$$

Manuscript received July 9, 1979; revised December 5, 1980 and November 16, 1981. Paper recommended by A. N. Michel, Past Chairman of the Stability, Nonlinear, and Distributed Systems Committee. This work was supported in part by the Office of Naval Research under Contract N00014-76-C-0017, and in part by the National Science Foundation under a Graduate Research Fellowship.

The authors are with the Center for Systems Science, Electrical Engineering Program, Yale University, New Haven, CT 06520. has received a great deal of attention in the control literature and the principal results of this theory are influencing the direction of research on general nonlinear systems. When the control $u(t)$ in (3) is a linear function of the state variables, (3) becomes a special case of (2), and the stability properties of such systems are bound to be of interest to control theorists.

This paper presents necessary and sufficient conditions for stability in the large of system (1) and asymptotic stability in the large of system (2). A brief treatment of some interesting properties of general homogeneous differential equations in Section II provides the setting for the results on pure quadratic systems developed in Section III. A simple statement of conditions for the stability of system (1) is given by Theorem 1. While the qualitative behavior of (1) can be completely characterized using a few simple prototypes (either with the aid of a heavy mathematical arsenal [2], [10], [12] or without [6], [9]), the addition of the linear part in (2) results in much more varied behavior. A simple classification of this system will not be complete; a complete classification will not be simple. Consequently, we limit our interest to one important aspect of the stability behavior of (2)-asymptotic stability in the large (a.s.1.). Within this restricted scope, a succinct statement of necessary and sufficient conditions is possible, as given in Theorem 2 at the beginning of Section IV, comprising the central result of this paper. However, it will be seen in the remainder of that section that a complete proof of this result involves the consideration of a number of special cases. These are explicitly listed in Theorem 3 (shown to be equivalent to Theorem 2) and their logical interrelation is depicted in Fig. 10, at the end of Section IV.

After this paper had been submitted for publication, a reviewer pointed out the existence of a paper by Dickson and Perko [3] on bounded planar quadratic systems. This interesting work derives necessary and sufficient conditions for system (2) to admit only bounded solutions, proceeding from the classification of system (1) given by Markus [10]. While that effort falls within the tradition of the historical literature cited above (listing sets of algebraic conditions on the coefficients of each particular case of a canonical parameterization in a specified coordinate system), our problem definition and methodology are quite different and slanted toward the interests of control theory. This paper presents a unified statement of conditions for global convergence involving familiar characteristics of $2 \times 2$ matrices, derived, free of coordinates, from simple topological properties of $B(x)$ and $f(x)$. The methodology 
encourages extensions of these results to systems of higher order and degree, and to other problems in nonlinear systems theory, such as controllability or stabilizability.

\section{Homogeneous Systems of Even Degree}

Consider the dynamical system in $\mathbb{R}^{n}$

$$
\dot{x}=h(x)
$$

where $h$ is an analytic function so that for each initial condition, $x_{0} \in \mathbb{R}^{n}$, there exists a unique solution, $p\left(t ; x_{0}\right)$, $t \geqslant 0$, satisfying (4). We assume $h(0)=0$, and adopt the following terminology. The set $U_{0 \leqslant t<\infty} p\left(t ; x_{0}\right)$ is a trajectory of system (4)-a smooth curve in $\mathbb{R}^{n}$. The system is said to be stable if for every $\epsilon>0$ there exists a $\delta>0$ such that $\left\|x_{0}\right\|<\delta$ implies $\left\|p\left(t ; x_{0}\right)\right\|<\epsilon, \forall t \in \mathbb{R}^{+}$. The origin is attractive if it is contained in some open neighborhood, $\mathscr{\pi}$, such that for all $x_{0} \in \Re$, for every $\epsilon>0$, there exists a $T \in \mathbb{R}^{-}$such that $\left\|p\left(t ; x_{0}\right)\right\|<\epsilon$ for $t>T$. The set $\pi$ is called the domain of attraction. System (4) is said to be asymptotically stable if it is stable and the origin is attractive. The system is said to be stable in the large if it is stable and all solutions are bounded. Finally, the system is said to be asymptotically stable in the large (a.s.1.) ${ }^{1}$ if it is asymptotically stable and the domain of attraction is the entire space, $\mathbb{R}^{n}$.

If $h$ is a homogeneous function, that is, $h(\beta x)=\beta^{k} h(x)$, $\beta \in \mathbb{R}, k \in \mathbb{N}$, then a well-known and useful fact concerning the solutions of (4) is given by the following lemma.

Lemma 2.1: Let $p\left(t ; x_{0}\right)$ be the solution of $(4)$ with the initial condition $p\left(0, x_{0}\right)=x_{0}$. Then for all $\beta \in \mathbb{R}$,

$$
p\left(t ; \beta x_{0}\right)=\beta p\left(\beta^{k-1} t ; x_{0}\right) .
$$

Then

Proof: Let $t=\beta^{k-1} s$ and define $u(s) \triangleq \beta p\left[t(s) ; x_{0}\right]$.

$$
\begin{aligned}
\frac{d}{d s} u(s) & =\beta \frac{d}{d t} p\left(t ; x_{0}\right) \frac{d t}{d s}=\beta^{k} \frac{d}{d t} p\left(t ; x_{0}\right) \\
& =h(u) .
\end{aligned}
$$

Hence, $u(s)$ satisfies (4) with initial condition $u(0)=\beta x_{0}$, as does $p\left(t ; \beta x_{0}\right)$, which implies $u(s)=p\left(t ; \beta x_{0}\right)$.

If $k$ is even, then the direction of the field in system (4) is constant along any straight line through the origin. In this case, as a direct result of Lemma 2.1 we have $p\left(t ;-x_{0}\right)=$ $-p\left(-t ; x_{0}\right)$. In other words, any trajectory through $x_{0}$ for $t \geqslant 0$ has an associated trajectory through $-x_{0}$ for $t \leqslant 0$, which is its reflection. This simple fact, depicted in Fig. 1, leads to the following corollary.

Corollary 2.1: If $k$ is even and the origin is stable, then for any $x_{0} \neq 0$ the complete trajectory $\gamma\left(x_{0}\right) \triangleq\left\{p\left(t ; x_{0}\right) \mid t\right.$ $\in \mathbb{R}\}$ is a positive distance from the origin.

Proof: Let $\left\|x_{0}\right\|>\epsilon>0$, but let the positive trajectory not be bounded away from the origin. Then there exists a sequence $\left\{t_{n}\right\}_{n \geq 1}, t_{n}>0$, such that $p\left(t_{n} ; x_{0}\right)<1 / n$. Defining $x_{n} \triangleq p\left(t_{n}, x_{0}\right)$ we have $p\left(t_{n} ;-x_{n}\right)=-x_{0}$ from

'Often called "global asymptotic stability."

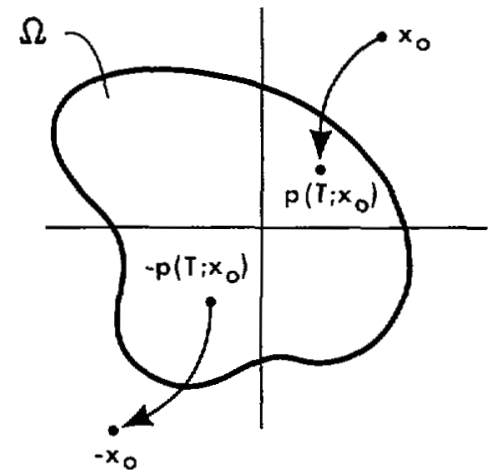

Fig. 1. Reflection property of even homogeneous systems.

Lemma 2.1. Hence, for no $n>0$ does $\left\|x_{n}\right\|<1 / n$ imply $\left\|p\left(t ;-x_{n}\right)\right\| \leqslant \epsilon$ and the origin is unstable. A similar argument applies to the negative trajectory.

In general, Corollary 2.1 indicates that an even degree homogeneous dynamical system in $\mathbb{R}^{n}$ can never be asymptotically stable. On the plane, $\mathbb{R}^{2}$, we may say even more. Equation (1) is a specific example of (4) in $\mathbb{R}^{2}$ for which $k=2$. The remainder of this paper will be devoted to the study of (1) and (2) in $\mathbb{R}^{2}$.

Lemma 2.2: No solution of system (1) other than an equilibrium point can be a closed path.

Proof: This follows directly from the fact that any nontrivial closed curve will intersect some line through the origin at least twice. A trajectory on this curve would imply that the field changes direction along that line which violates the even homogeneous property of (1).

Corollary 2.2: If the origin of system (1) is stable, then the field must vanish along at least an entire line through the origin.

Proof: It suffices to show that an equilibrium state $x_{0} \neq 0$ exists. By homogeneity the conclusion follows.

Assume that the system is stable, and let $\gamma \not \equiv 0$ be a trajectory of (1) contained in some compact neighborhood of 0 . From Corollary 2.1, $0 \notin \bar{\gamma}$ (where $\bar{\gamma}$ is the closure of $\gamma$ ). If $\bar{\gamma}-\gamma$ is not an equilibrium point, then by the Poincare-Bendixson theorem [5, p. 54] either $\gamma$ is a limit cycle or its positive limit set is a limit cycle. But this contradicts Lemma 2.2. Hence, $x_{0} \triangleq \bar{\gamma}-\gamma$ is an equilibrium point and $B\left(x_{0}\right)=0$. Since $x_{0} \neq 0$, we have $B(x)=0$ for all $x=\alpha x_{0}, \alpha \in \mathbb{R}$, by homogeneity.

The next section will investigate the existence of lines along which the field in (1) vanishes as required by the previous corollary, leading to a new parameterization of stable quadratic systems. This new parameterization allows the characterization of stability behavior in terms of a matrix in $\mathbb{R}^{2 \times 2}$, a result whose consequences pervade the remainder of this paper.

\section{STability of Second-Order Quadratic SYSTEMS}

In this section we shall exclusively consider the particular class of second-degree second-order systems described by (1): 


$$
\dot{x}=\left[\begin{array}{l}
x^{T} G x \\
x^{T} H x
\end{array}\right] \triangleq B(x) .
$$

We assume that at least one of either $G$ or $H \in \mathbb{R}^{2 \times 2}$ is nonzero and, without loss of generality, that both are symmetric. From Corollary 2.2 it follows that the locus of the set of critical points of $B(x)$ is crucial to the stability properties of (1). This is completely determined by $G$ and $H$, and using familiar properties of symmetric matrices, we may classify the types of equilibrium states of (1) according to whether the field vanishes, as follows:

1) only at the origin,

2) along a straight line through the origin,

3) along two straight lines through the origin.

It may be noted that as an immediate consequence of Corollary 2.2, systems of type 1) cannot be stable. In cases 2) and 3) the quadratic forms, $x^{T} G x$ and $x^{T} H x$, in (1) share a common real linear factor. These properties lead to the following theorem which represents the central result of this section.

Theorem I: System (1) is stable in the large if and only if there is some constant vector $c \in \mathbb{R}^{2}$ and a real constant matrix $D \in \mathbb{R}^{2 \times 2}$ with complex conjugate eigenvalues such that

$$
B(x)=c^{T} x D x
$$

The remainder of this section is devoted to the proof of Theorem 1.

\section{A. Notation and Definitions}

Since indefinite and semidefinite matrices will arise in all subsequent discussions, it is worth establishing the following notational conventions concerning their algebraic and geometric properties. Let $A_{s}$ denote the symmetric part of $A$. The set $\delta[A] \triangleq\left\{M \mid A_{s}=M_{s}\right\}$ denotes the symmetric equivalence class of $A$. Clearly, $x^{T} M x=x^{T} A x \forall x \in \mathbb{R}^{2}$ if and only if $M \in \mathcal{S}[A]$. If $A$ is singular, then for some $a, b \in \mathbb{R}^{2}$ we have $A=a b^{T}$. Hence, for any $P \in S[A], x^{T} P x$ $=0$ iff $x$ is orthogonal to either $a$ or $b$.

The class $\delta[0]$ contains a matrix $J$ with the property $J^{2}=-I . J$ is the skew-symmetric matrix $\left[\begin{array}{rr}0 & -1 \\ 1 & 0\end{array}\right]$ and maps every vector in $\mathbb{R}^{2}$ into $x_{\perp} \triangleq J x$, a vector in the orthogonal complement of $x$. From these definitions it follows that 1) $x^{T} x_{\perp}=0, \forall x \in \mathbb{R}^{2}$; 2) $x^{T} y_{\perp}=0$ iff $x=\alpha y$, $\alpha \in \mathbb{R}$; 3) $x^{T} y_{\perp}=x^{\frac{T}{T}} J y=|y, x|$, where the last symbol denotes the determinant of the array $[y, x]$.

We denote the subspace defined by $x \in \mathbb{R}^{2}$ as $(x) \triangleq\{y \mid y$ $=\alpha x, \alpha \in \mathbb{R}\}$, and its orthogonal complement as $\left(x_{\perp}\right) \triangleq\{y \mid$ $\left.y^{T} x=0\right\}$.

Finally, if $\left(x_{0}\right)$ is a nonzero fixed direction of $B(x)$-i.e., $0 \neq B\left(x_{0}\right) \in\left(x_{0}\right), x_{0} \neq 0$-then $\left(x_{0}\right)^{+}$and $\left(x_{0}\right)^{-}$[the positive and negative rays contained in the line $\left.\left(x_{0}\right)\right]$ are said to be ray solutions of system (1). It is worth noting in passing that a positive ray solution of (1) must have a finite escape time-i.e., for some $T<\infty, \lim _{t \rightarrow T} p\left(t ; x_{0}\right)=\infty$ [6].

\section{B. Quadratic Differential Equations with Nonisolated Equilibria}

We have shown that (1) is unstable if it is of type 1) in the introduction of this section (in fact, such systems must have ray solutions as shown in [2], [6], [10]). Accordingly, we need only consider systems of type 2) and 3)-systems with at least one line of equilibrium states passing through the origin. The following lemma provides a useful characterization of such systems.

Lemma 3.1: System (1) is type 2) or 3) if and only if there is some $c \in \mathbb{R}^{2}$ and $D \in \mathbb{R}^{2 \times 2}$ such that $B(x)=c^{T} x D x$.

Proof: $B(x)$ is type 2) or 3 ) if and only if for some $y \neq 0$

$$
B(y)=\left[\begin{array}{c}
y^{T} G y \\
y^{T} H y
\end{array}\right]=0 .
$$

Now $y^{T} G y=0$ if and only if $G \in \delta\left[d_{1} c^{T}\right]$ for some $c \in\left(y_{\perp}\right]$ and $d_{1} \in \mathbb{R}^{2}$. Similarly, $H \in \mathcal{S}\left[d_{2} c^{T}\right]$ for some $d_{2} \in \mathbb{R}^{2}$. Then, defining

$$
D \triangleq\left[\begin{array}{c}
d_{1}^{T} \\
-d_{2}^{T}
\end{array}\right]
$$

we have

$$
B(x)=\left[\begin{array}{c}
x^{T} G x \\
x^{T} H x
\end{array}\right]=\left[\begin{array}{l}
\left(x^{T} c\right)\left(d_{1}^{T} x\right) \\
\left(x^{T} c\right)\left(d_{2}^{T} x\right)
\end{array}\right]=c^{T} x D x .
$$

The importance of this lemma is that it relates individual solutions of a nonlinear time invariant system to solutions of a linear time-varying system. Since $d x / d t=c^{T} x D x$, if $d s / d t \triangleq c^{T} x$, then

$$
\frac{d x}{d s}=D x(s)
$$

Thus, if $p\left(t ; x_{0}\right)$ is a solution to system (1) of type 2$)$ or 3 ), then

$$
p\left(t ; x_{0}\right)=e^{s(t) D} x_{0} \quad s(t)=\int_{0}^{t} c^{T} p\left(\tau ; x_{0}\right) d \tau
$$

and that trajectory remains on the integral curve of system (5) specified by $x_{0}$ with position parameterized by $s(t)$. In consequence of the homogeneity property of (1) discussed in Section II, a trajectory in the half-plane $c^{T} x>0$ (or $c^{T} x<0$ ) never leaves that half-plane, hence if $c^{T} x_{0}>0$ then $s(t)>0 \forall t \in \mathbb{R}^{+}$(or if $c^{T} x_{0}<0$, then $s(t)<0 \forall t \in \mathbb{R}^{+}$).

\section{Necessary and Sufficient Conditions for Stability in the Large}

By reparameterizing (1) as (5) [for types 2) and 3)] we are able to introduce well-known properties of planar linear systems in the proof of Theorem 1. Fig. 2 depicts the established catalog of integral curves of (5) for $D$ not identically zero [5]. 


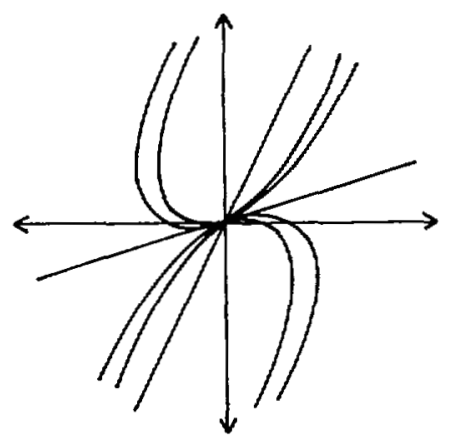

(a)

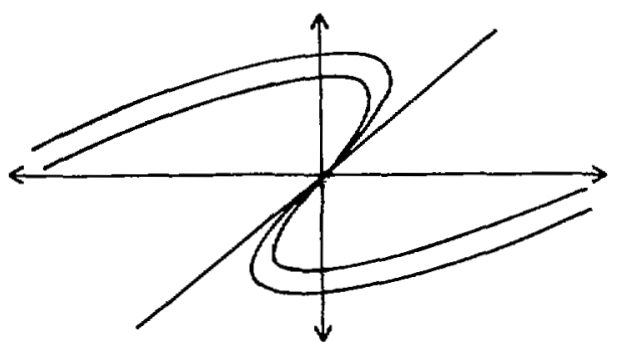

(d)

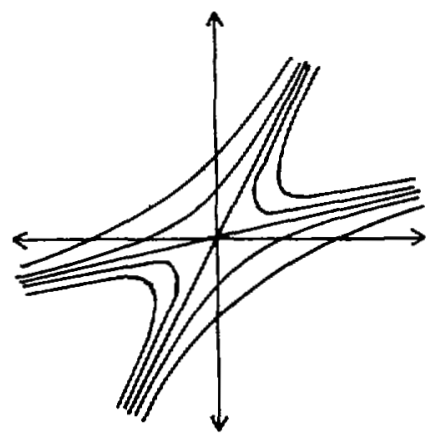

(b)

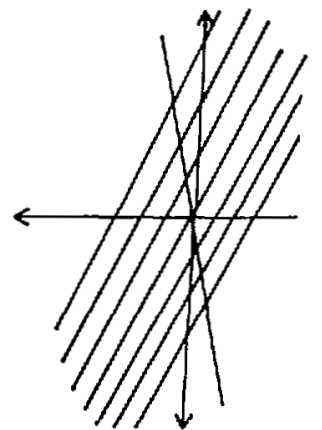

(e)

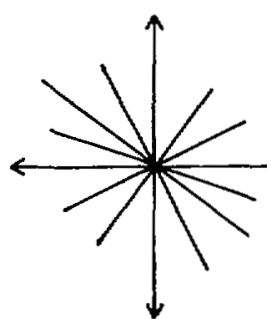

(c)

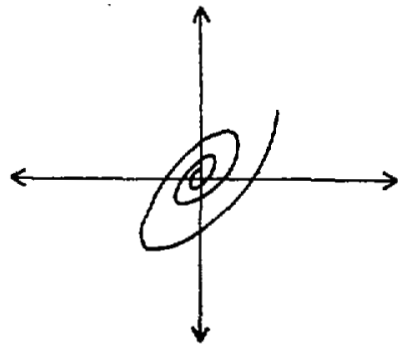

(f)

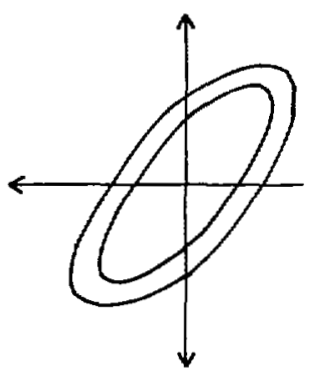

(g)

Fig. 2. Integral curves of planar linear systems. (a) Node. (b) Saddle. (c) Improper node. (d) Partial node. (e) Singular system matrix. (f) Focus. (g) Center.

If system (1) is type 3)-i.e., has two distinct lines of equilibria-then $D$ must be singular, and the system trajectories lie on curves depicted in Fig. 2(e). In this case $D=a b^{T}$ where $b \notin(c)$; hence $B(x) \in(a)$, and $B(x)=0$ on $\left(b_{\perp}\right)$ and $\left(c_{\perp}\right)$. If $(a) \neq\left(c_{\perp}\right)$ and $(a) \neq\left(b_{\perp}\right)$, then $(a)$ contains a positive ray solution of (1) [as depicted in Fig. 3(a)] according to Lemma 2.1. If $(a)=\left(c_{\perp}\right)$, then any trajectory directed toward $\left(b_{\perp}\right)$ has a reflected trajectory directed away from $\left(b_{\perp}\right)$ [as depicted in Fig. 3(b)], again by Lemma 2.1. In either case there are unbounded solutions of (1) for initial conditions arbitrarily close to the origin, and the system is not stable.

If system (1) is type 2)-i.e., has a single line of equilibria given by $\left(c_{\perp}\right)$ - and $D$ has real eigenvalues, then the system trajectories lie on curves depicted in Fig. 2(a)-(e). In the cases 2(a)-(d) there is at least one trajectory, $\gamma$, whose closure intersects $\left(c_{\perp}\right)$ only at the origin, regardless of the choice of $c$. Thus by Lemma 2.1 either $\gamma$ or $-\gamma$ tends to infinity with increasing $t$. In the case depicted by
Fig. 2(e) the same statement holds except when $\left(c_{\perp}\right)$ is parallel to the trajectory lines, in which case every nonequilibrium solution tends to infinity. Typical solutions of system (1) are depicted in Fig. 3(c)-(e), corresponding to the case that system (5) is a node, partial node, or $D$ is singular, respectively. In all these cases, system (1) is unstable.

Since these cases exhaust all instances of system (1), type 2 ) and type 3 ) when $D$ has real eigenvalues, and since type 1) was shown to be unstable earlier, we have proven the following proposition.

Proposition 3.1: System (1) is unstable if it is of type 1) or if for some $c \in \mathbb{R}^{2}$, and $D \in \mathbb{R}^{2 \times 2}, B(x)=c^{T} x D x$ and $D$ has real eigenvalues.

This is evidently a restatement of the necessary conditions of Theorem 1 . We now proceed to show that the conditions of Theorem 1 are sufficient as well.

If system (1) is type 2 ) and $D$ has complex conjugate eigenvalues, then system trajectories lie on curves depicted 


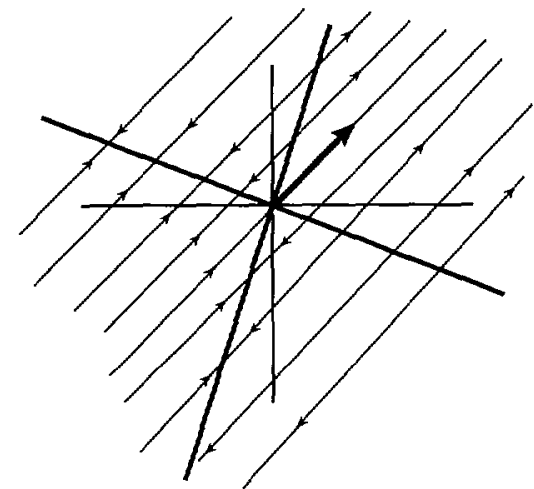

(a)

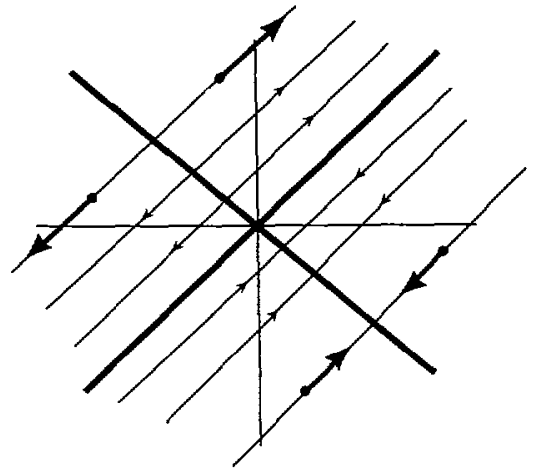

(b)

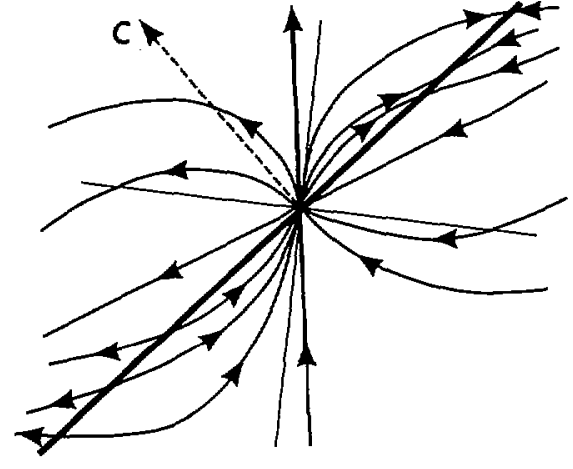

(c)

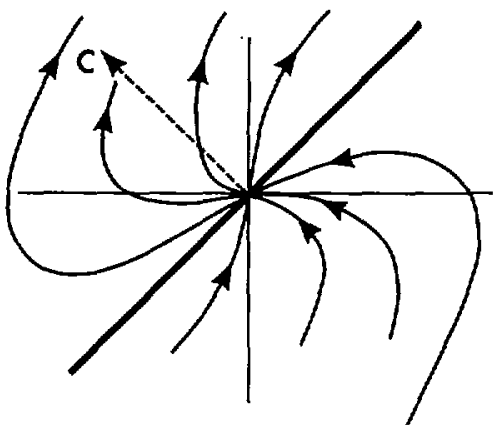

(d)

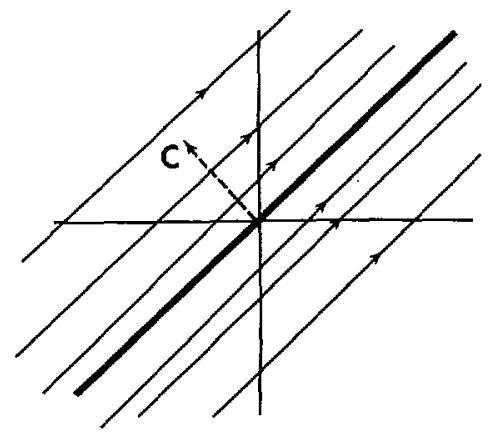

(e)

Fig. 3. Typical unstable solutions of system (1) where $B(x)=c^{T} x D x$. (a) Type 3) with a ray solution. (b) Type 3) with no ray solution. (c) Type 2) with a ray solution. (d) Type 2) with no ray solution. (c) Type 2) with singular matrix $D$.

in Fig. 2(f), (g). Since the half-planes $c^{T} x>0$ and $c^{T} x<0$ are positive-invariant by homogeneity, and every segment of the curves in $2(\mathrm{f}),(\mathrm{g})$ intersect $\left(c_{\perp}\right)$ at a finite point, all trajectories are bounded, and $s(t) \in\left[T_{1}, T_{2}\right], 0<T_{1}<T_{2}<$ $\infty$, for $t \in \mathbb{R}^{+}$when $c^{T} x_{0}>0$ (with a corresponding statement for $\left.c^{T} x_{0}<0\right)$. Hence, $\left\|p\left(t ; x_{0}\right)\right\|=\left\|e^{s(t) D} x_{0}\right\| \leqslant$ $\sup _{t \in\left[T_{1}, T_{2}\right]}\left\|e^{i D}\right\| \cdot\left\|x_{0}\right\| \leqslant \rho\left\|x_{0}\right\|$ for some $\rho \in \mathbb{R}^{+}$and the system is stable. Since this argument is independent of $\left\|x_{0}\right\|$, the system is stable in the large. Analog computer simulations of stable quadratic systems are plotted in Fig. 4. We have thus proven the following.

Proposition 3.2: System (1) is stable in the large if there exist a $c \in \mathbb{R}^{2}$ and $D \in \mathbb{R}^{2 \times 2}$ such that

$$
B(x)=c^{T} x D x
$$

and $D$ has complex conjugate eigenvalues.

This is a restatement of the sufficient conditions of Theorem 1. Therefore, taken together, Propositions 3.1 and 3.2 constitute the proof of that theorem.

\section{Summary}

The results of this section indicate that most second-order quadratic differential equations are unstable: only those systems whose solutions lie on the integral curves of a linear system whose equilibrium state is a center or a focus may be stable. An exhaustive account of the qualitative behavior of the far more pervasive unstable examples of system (1) is given in [2], [6], [9], [10], [12]. Since this paper is concerned solely with questions of stability, we are content here to ignore those results and immediately apply the results of Theorem 1 to the problem of a.s.l. for system (2).

\section{ASYMPTOTIC STABILITY IN THE LARGE OF SYSTEM (2): $\dot{x}=A x+B(x)$}

As mentioned in the Introduction, the addition of the linear part in (2),

$$
\dot{x}=A x+B(x) \triangleq f(x),
$$

results in such diversity of behavior that we confine our attention exclusively to the question of asymptotic stability in the large (a.s.1.). This is completely resolved by Theorem 2 , which represents the central contribution of the paper.

Theorem 2: The following conditions are necessary and sufficient for the asymptotic stability in the large of system (2):

1) the eigenvalues of $A$ have nonpositive real parts;

2) there exist a $c \in \mathbb{R}^{2}$ and a $D \in \mathbb{R}^{2 \times 2}$ such that $B(x)=c^{T} x D x$

3) the pencil $A+\mu(x) D$, where $\mu(x) \triangleq-|A x, x| /$ $|D x, x|$, has nonpositive eigenvalues for all $x \in \mathbb{R}^{2}$ where it is defined; is undefined, if ever, only on an eigenvector of $A$ in the null space of $D$ and $|A| \neq 0$; is singular on, at most, a unique line, $\left(x_{0}\right)$, and $c_{\perp} \in\left(x_{0}\right)$ iff $|A| \neq 0$.

Roughly speaking, conditions 1) and 2) of this theorem correspond to the requirement that both the linear and the quadratic part of (2) not be unstable when considered 


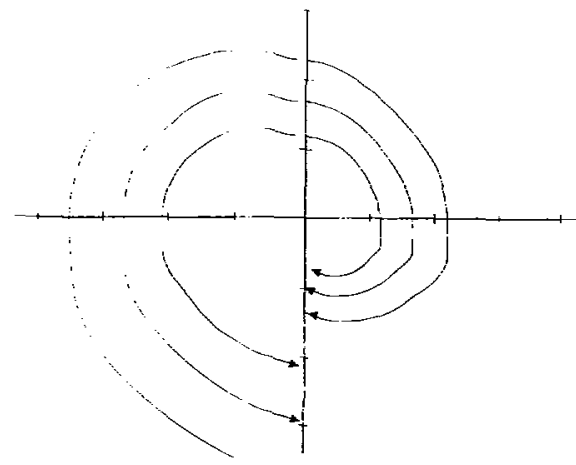

(a)

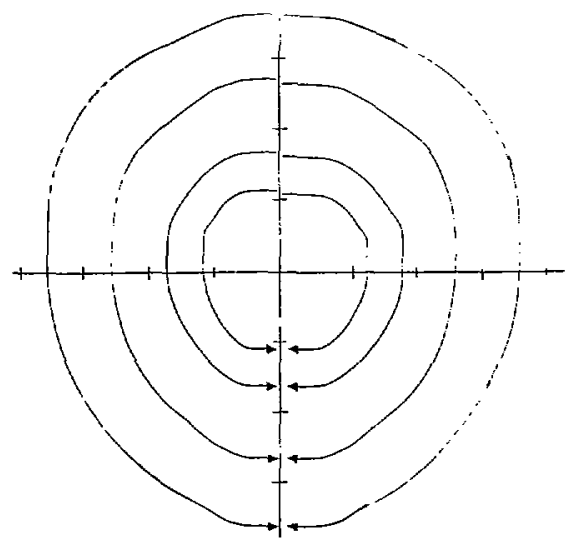

(b)

Fig. 4. Analog computer simulations of stable pure quadratic systems (a) Focus behavior: $\dot{x}=x_{1}\left[\begin{array}{rr}0 & 1 \\ -0.76 & -0.30\end{array}\right] x$. (b) Center behavior: $\dot{x}=x_{1}\left[\begin{array}{rr}0 & 1 \\ -1 & 0\end{array}\right] x$.

alone. Condition 3), which depends upon the ratio of two determinants involving $A$ and $D$ (refer to notation introduced in Section III-A), will be seen to ensure that the origin is the unique equilibrium state of system (2), and that the perturbation on the quadratic part of the field introduced by $A x$ is always directed toward that equilibrium state.

While the restricted question of a.s.l. behavior does permit a relatively succinct characterization as given above, the complexity of this system is such that several distinct cases arise in the course of proving these results. For ease of exposition, we prefer to prove Theorem 2 by treating an equivalent statement, given as Theorem 3, below. This reformulation is essentially a translation of condition 3 ) in terms of well-known properties of $2 \times 2$ matrices. While it results in a less concise statement, Theorem 3 affords a more natural framework for treating the various special cases as they arise. Moreover, it is obviously of greater practical use, since it specifies an easy numerical check for the stability properties of (2).

In order to facilitate future discussion, we introduce the following conventions. We will call a real $2 \times 2$ matrix focal if it has a pair of complex conjugate eigenvalues; $x$-critical if it has a unique one-dimensional eigenspace given by $(x)$; and nodal if it has at least two distinct real eigenvectors.
For example, $\left[\begin{array}{rr}1 & 1 \\ -1 & 1\end{array}\right]$ is focal, $\left[\begin{array}{ll}1 & 1 \\ 0 & 1\end{array}\right]$ is $x$-critical where $x \triangleq\left[\begin{array}{l}1 \\ 0\end{array}\right]$, and $\left[\begin{array}{ll}1 & 0 \\ 0 & 1\end{array}\right]$ is nodal. Note that a singular matrix may be either nodal (e.g., $\left[\begin{array}{ll}1 & 0 \\ 0 & 0\end{array}\right]$ ) or $x$-critical (e.g., $\left[\begin{array}{ll}0 & 1 \\ 0 & 0\end{array}\right]$ ). Recall from Section III-A that $J$ $\triangleq\left[\begin{array}{rr}0 & -1 \\ 1 & 0\end{array}\right]$ is the skew-symmetric matrix in $\mathbb{R}^{2}$. With these definitions in force, Theorem 2 may be restated as follows.

Theorem 3: System (2) is a.s.l. if and only if conditions 1) and 2) of Theorem 2 hold and

a) $[J D]_{s}$ and $\left[D^{T} J A\right]_{s}$ are definite or semidefinite and have the same sign;

b) one of the following mutually exclusive conditions holds:

b1) $D$ and $D^{-1} A$ are both focal.

b2) $D$ is focal and $D^{-1} A$ is $x$-critical where $x \in\left(c_{\perp}\right)$ iff $|A| \neq 0$.

b3) $D$ is $x$-critical, singular, $|A| \neq 0$, and $A^{-1} D=\gamma D$ for some scalar $\gamma \neq 0$.

To clarify the significance of these conditions we will give a few representative examples of (2) along with analog computer simulations. Example 4.1 consists of two a.s.l. systems with a stable, but not asymptotically stable linear part, motivating the wording of condition 1).

Example 4.Ia [ Fig. 5(a)]:

$$
\begin{aligned}
& \dot{x}_{1}=x_{2}+.1 x_{1}\left(x_{1}+x_{2}\right) \\
& \dot{x}_{2}=-x_{1}+.1 x_{1}\left(x_{2}-x_{1}\right)
\end{aligned}
$$

Example 4.1b [Fig. 5(b)]:

$$
\begin{aligned}
& \dot{x}_{1}=-x_{1}-x_{2}\left(x_{1}+x_{2}\right) \\
& \dot{x}_{2}=x_{1}\left(x_{1}+x_{2}\right) .
\end{aligned}
$$

Since $|D x, x|=x^{T} J D x$ (see Section III-A), condition a) evidently requires that $D$ not be nodal. However, unlike the situation in Section III, it is not necessary that $D$ be focal: condition b3) indicates that a special class of unstable quadratic systems may give rise to a.s.l. behavior in (2). Example 4.2 presents such a system.

Example 4.2 [Fig. 5(c)]:

$$
\left[\begin{array}{l}
\dot{x}_{1} \\
\dot{x}_{2}
\end{array}\right]=\left[\begin{array}{rr}
-1 & 0 \\
0 & -1
\end{array}\right]\left[\begin{array}{l}
x_{1} \\
x_{2}
\end{array}\right]+x_{1}\left[\begin{array}{ll}
0 & 0 \\
1 & 0
\end{array}\right]\left[\begin{array}{l}
x_{1} \\
x_{2}
\end{array}\right] .
$$

Since $|D| \cdot\left|D^{-1} A x, x\right|=|A x, D x|=x^{T} D^{T} J A x$ (again, see Section III-A), condition a) also excludes the case where $D^{-1} A$ is nodal. It will be seen that eigenvectors of $D^{-1} A$ generally specify subspaces in $\mathbb{R}^{2}$ containing additional equilibrium states, which must be excluded for the origin to be a.s.1. However, condition b2) indicates that in special cases, an eigenvector of $D^{-1} A$ may not result in additional equilibria, and a.s.l. may occur. Example 4.3 presents two such systems: $D^{-1} A$ is critical in both cases and singular in 4.3b. 
Example 4.3 [Fig. $5(d),(b)$, respectively]:
a) $\dot{x}=\left[\begin{array}{ll}-1 & 1 \\ -1 & 0\end{array}\right] x+x_{1}\left[\begin{array}{rr}0 & -1 \\ 1 & 0\end{array}\right] x$
b) $\dot{x}=\left[\begin{array}{rr}-1 & 0 \\ 0 & 0\end{array}\right] x+\left(x_{1}+x_{2}\right)\left[\begin{array}{rr}0 & -1 \\ 1 & 0\end{array}\right] x$.

The sign agreement stipulated in condition a) of Theorem 3 will be seen to imply that the linear part of the field perturbs the quadratic part in such a fashion that solutions of (2) cross the integral curves of (1) in the direction of the origin at every point on the plane. This is equivalent to the sign condition on the eigenvalues of the pencil, $A+\mu(x) D$, given in Theorem 2, and is crucial for a.s.l. Example 4.4 presents a system where $A$ is asymptotically stable, $D$ is focal so that the pure quadratic field is stable, $D^{-1} A$ is focal so that there are no equilibrium states other than the origin, but this sign condition fails to hold; unbounded solutions result.

Example 4.4 [Fig. 5(e)]:

$$
\left[\begin{array}{l}
\dot{x}_{1} \\
\dot{x}_{2}
\end{array}\right]=\left[\begin{array}{rr}
-1 & 1 \\
-1 & -1
\end{array}\right]\left[\begin{array}{l}
x_{1} \\
x_{2}
\end{array}\right]+x_{1}\left[\begin{array}{rr}
2 & -1 \\
1 & 2
\end{array}\right]\left[\begin{array}{l}
x_{1} \\
x_{2}
\end{array}\right] .
$$

Condition b1) should be seen as the typical case of a.s.l. behavior of system (2), two examples of which are given below.

Example 4.5 [Fig. 5(f), $(g)$, respectively]:
a) $\dot{x}=\left[\begin{array}{rr}-1 & 0 \\ 0 & -1\end{array}\right] x+x_{1}\left[\begin{array}{rr}0 & -1 \\ 1 & 0\end{array}\right] x$
b) $\dot{x}=\left[\begin{array}{rr}-1 & 2 \\ -2 & -1\end{array}\right] x+x_{1}\left[\begin{array}{rr}1 & 1 \\ -1 & 1\end{array}\right] x$.

After establishing some useful preliminary results in Section IV-A, we will prove the necessity of the conditions listed under Theorem 3 in Section IV-B, prove their sufficiency in Section IV-C, and finally, prove the equivalence of Theorems 2 and 3 by way of summary in Section IV-D. The logical interrelation between the various cases considered in the sequel is depicted in Fig. 10 at the end of this section.

\section{A. Discussion and Mathematical Preliminaries}

In this section we derive several results which will be widely used in subsequent proofs.

The existence of a positive-invariant set disconnected from the origin is a sufficient condition for the equilibrium state of system (2) not to be globally asymptotically stable. A systematic study of conditions for the existence of such sets, using Propositions 4.1 and 4.2, leads to the necessary conditions of Section IV-B. Proposition 4.1 concerns the existence of an equilibrium state removed from the origin (hereafter referred to as an off-origin equilibrium), while Proposition 4.2 introduces a device used to demonstrate the existence of general positive-invariant sets.

Proposition 4.1: System (2) has an equilibrium state $x_{e}$ $\neq 0$ if and only if for some $x \in\left(x_{e}\right)$ and $x \neq 0$ either $A x=B(x)=0$ or $A x \neq 0, B(x) \neq 0$ and $B(x) \in(A x)$.
Proof: The proof follows directly from the fact that $x_{e}$ is an equilibrium state if and only if the field in system (2) vanishes at $x_{e}$.

If on the boundary of an open set in $\mathbb{R}^{2}$, the field is directed everywhere toward its interior, then that set is positive-invariant. Proposition 4.2, an extension of the well-known Chetaev instability theorem, establishes this fact formally and is stated without proof.

Proposition 4.2: Let $V$ be a continuously differentiable functional on $\mathbb{R}^{2}$, and let $\Omega \subseteq \mathbb{R}^{2}$ be a nonempty open set such that $V>0$ on $\Omega, V=0$ on $\partial \Omega$, the boundary of $\Omega$, and $\dot{V} \geqslant 0$ on $\Omega$. Then $\Omega$ is a positive-invariant set.

Throughout Section IV-B it will be convenient to use the following notation. If $P$ is symmetric, we will call $e_{\gamma} \triangleq\{x$ $\left.\in \mathbb{R}^{2} \mid x^{T} P x>\gamma\right\}$ the cone defined by $P$. Note that if $P=$ $\left[a b^{T}\right]_{s}$, then $\mathcal{C}_{0}(P)$ is a set of lines bounded by $\left(a_{\perp}\right)$ and $\left(b_{\perp}\right)$, hence we will sometimes refer to this cone as $e_{0}\left(a_{\perp}, b_{\perp}\right)$. Some useful results on cones are contained in Appendix A to which we will frequently refer in the sequel. Finally, by the half-plane of $c$ we shall intend $\mathcal{K}_{+}(c) \triangleq\{x$ $\left.\in \mathbb{R}^{2} \mid c^{T} x>0\right\}$.

In Section IV-C the sufficiency proof will involve the construction of positive-invariant regions around the origin. Proposition 4.3 and its corollary lay the foundation for these proofs as well as some of the argument of necessity by establishing a connection between eigenvalues of the pencil $A+\mu(x) D$, and the resultant sum of $A x$ and $D x$ in (2). This result is also the central link between Theorems 2 and 3.

Proposition 4.3: For any pair of matrices, $A, D \in \mathbb{R}^{2 \times 2}$, and any $x \in \mathbb{R}^{2}$, if $\mu(x) \triangleq-|A x, x| /|D x, x|$ is defined, then $x$ is an eigenvector of the pencil $A+\mu(x) D$ with corresponding eigenvalue $\lambda(x) \triangleq-|A x, D x| /|D x, x|$.

Proof: Define $\alpha(x) \triangleq|A x, x|$ and $\delta(x) \triangleq|D x, x|$. Since $|[\delta A-\alpha D] x, x|=\delta|A x, x|-\alpha|D x, x|=0$ for all $x \in \mathbb{R}^{2}$ it follows that $[\delta A-\alpha D] x=\eta(x) x$ for some real valued function $\eta$. But

$$
\begin{aligned}
x^{T} x \eta & =x^{T}[\delta A-\alpha D] x=\left|\begin{array}{ll}
x^{T} A x & x^{T} D x \\
x^{T} J A x & x^{T} J D x
\end{array}\right| \\
& =\left|\left[x, J^{T} x\right]^{T}[A x, D x]\right|=-x^{T} x|A x, D x|,
\end{aligned}
$$

hence $\eta(x)=-|A x, D x|$ and $\delta[A+\mu(x) D] x=\eta x$.

Corollary 4.1: If $D$ is focal and $D^{-1} A$ is not nodal, then the pencil $A+\mu(x) D$ has eigenvalues in $\overline{\mathbb{R}^{-}}$for all $x \in \mathbb{R}^{2}$ iff condition a) of Theorem 3 holds.

Proof: Since $|D x, x| \neq 0$ and hence sign definite, $\mu(x)$ and $\lambda(x)$ exist for all $x \in \mathbb{R}^{2}$. Since $|A x, D x|=|D|$ $\left|D^{-1} A x, x\right|, \lambda(x)$ is either strictly negative or strictly positive when $D^{-1} A$ is focal, and semidefinite, with a zero on $\left(x_{0}\right)$ if $D^{-1} A$ is $x_{0}$-critical. But $|D x, x|=x^{T} J D x$ and $A x, D x \mid=x^{T} D^{T} J A x$; hence $\lambda \leqslant 0$ for all $x \in \mathbb{R}^{2}$ iff $[J D]_{s}$ and $\left[D^{T} J A\right]_{s}$ agree in sign.

\section{B. Necessary Conditions for Asymptotic Stability in the Large}

This section is devoted to the proof that the conditions of Theorem 3 are necessary for (2) to be a.s.l. From the 


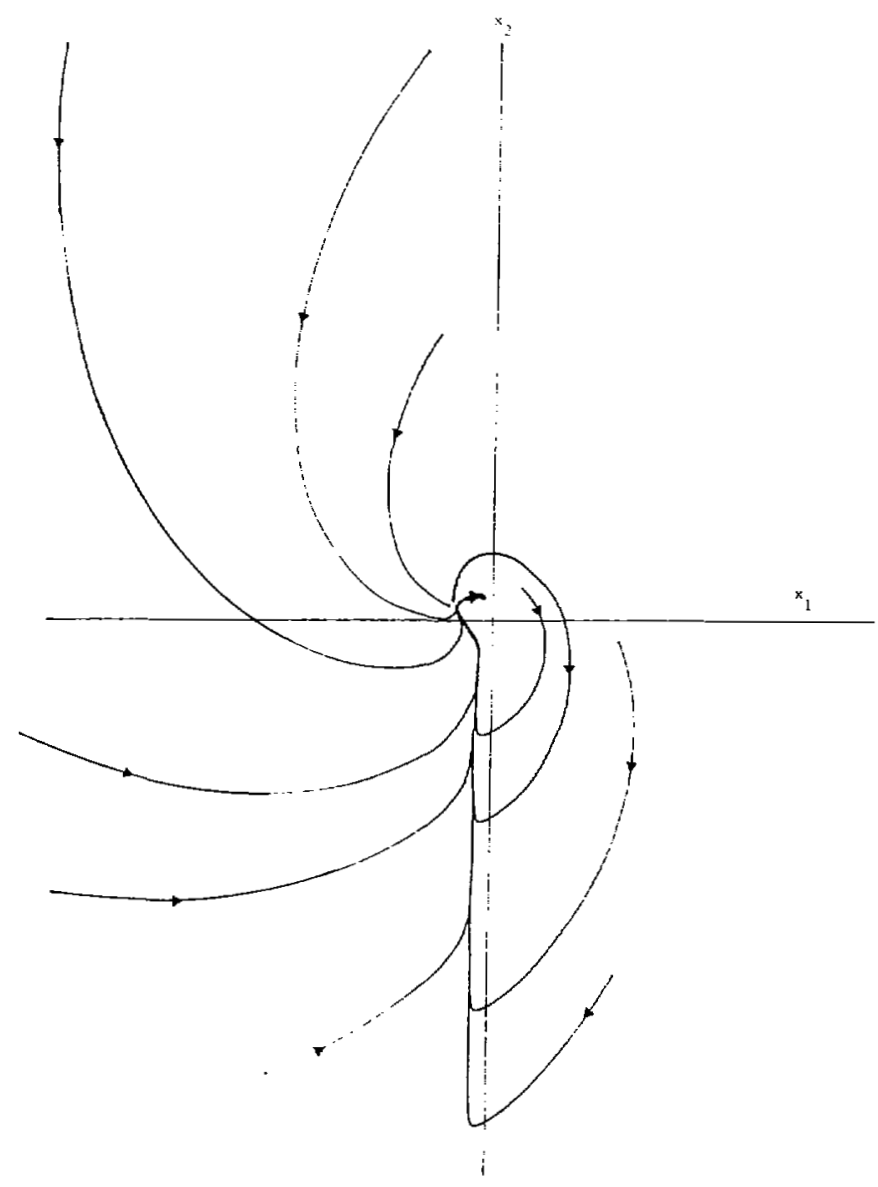

(a)

Fig. 5. Analog computer simulations of representative linear-quadratic systems. (a) a.s.l. system with linear part at center (Example 4.1 a).

results of Lyapunov it is known that a necessary condition for system (2) to be stable is that the spectrum of $A$ be contained in the closed left half of the complex plane, $\overline{\mathbb{C}}^{-}$, and this gives rise to condition 1) in Theorem 3. If the stability properties of the linear part of (2) generally determine local behavior, intuition suggests that its global behavior is determined by the quadratic part, $B(x)$. This is partially verified by the following four lemmas.

Lemma 4.I: If $|A| \neq 0$ and $B(x)$ is type 1 ), then system (2) has an off-origin equilibrium.

Proof: Since $x \neq 0$ implies $A x \neq 0$ and $B(x) \neq 0$ under the hypothesis above, it suffices to show that for some $x_{0} \neq 0, B\left(x_{0}\right) \in\left(A x_{0}\right)$ (by Proposition 4.1). Since $B(x) \in$ $(A x)$ iff $|A x, B(x)|=0$, let

$$
\begin{aligned}
A & =\left[\begin{array}{l}
a_{1}^{T} \\
a_{2}^{T}
\end{array}\right] \text { to get }|A x, B(x)| \\
& =\left|\begin{array}{ll}
a_{1}^{T} x & x^{T} G x \\
a_{2}^{T} x & x^{T} H x
\end{array}\right|=x_{1}^{3} q\left(x_{2} / x_{1}\right)
\end{aligned}
$$

where $q$ is a cubic polynomial whose leading coefficient is $a_{12} h_{22}-a_{22} g_{22} \triangleq \alpha$. If $\alpha \neq 0$, there exists a real root $v_{0}$ of

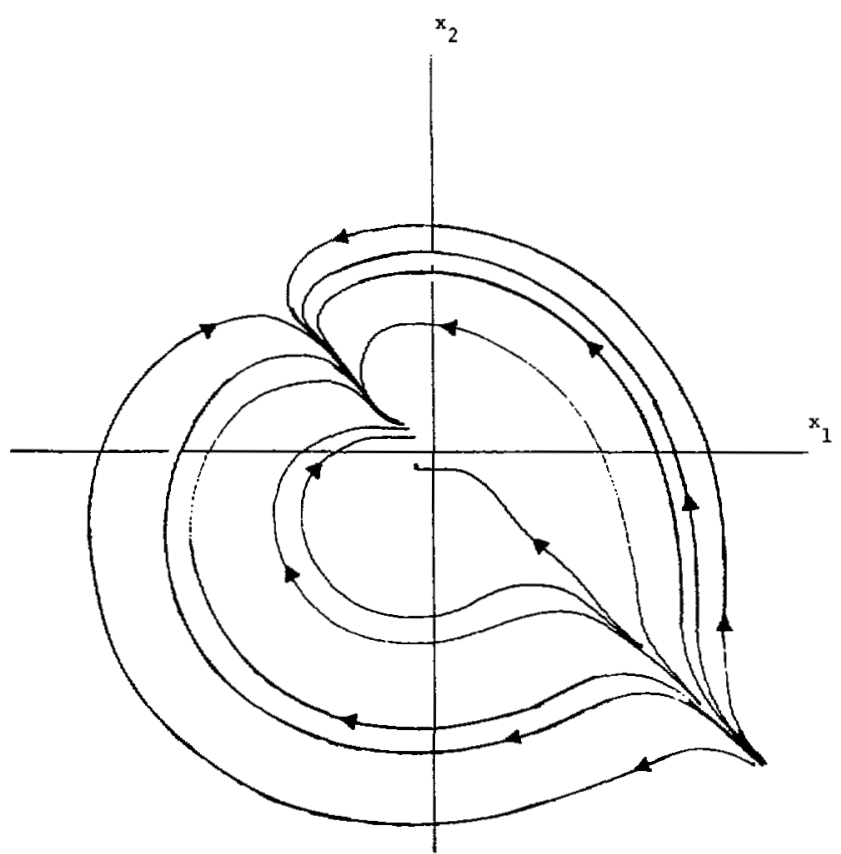

(b)

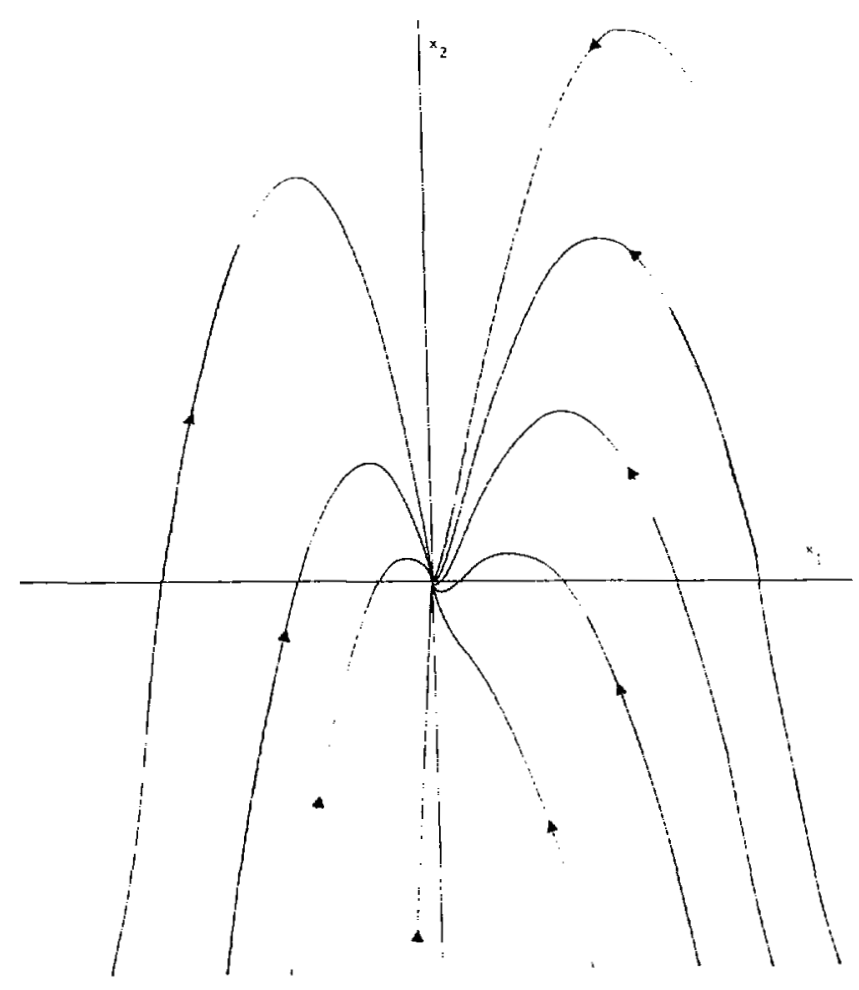

(c)

Fig. 5. (Continued.) (b) a.s.l. system with singular linear part (Examples $4.1 \mathrm{~b}$ and $4.3 \mathrm{~b}$ ). (c) a.s.1. system with $x$-critical $D$ matrix (Example 4.2).

$q(v)$ and hence $x_{0} \triangleq\left[\begin{array}{c}\beta \\ \beta v_{0}\end{array}\right]$ is an equilibrium point for some $\beta \in \mathbb{R}$. If $\alpha=0$, then for $x_{0} \triangleq\left[\begin{array}{l}0 \\ 1\end{array}\right]$, we have $\left|A x_{0}, B\left(x_{0}\right)\right|=\left|\begin{array}{ll}a_{12} & g_{22} \\ a_{22} & h_{22}\end{array}\right|=0$. 


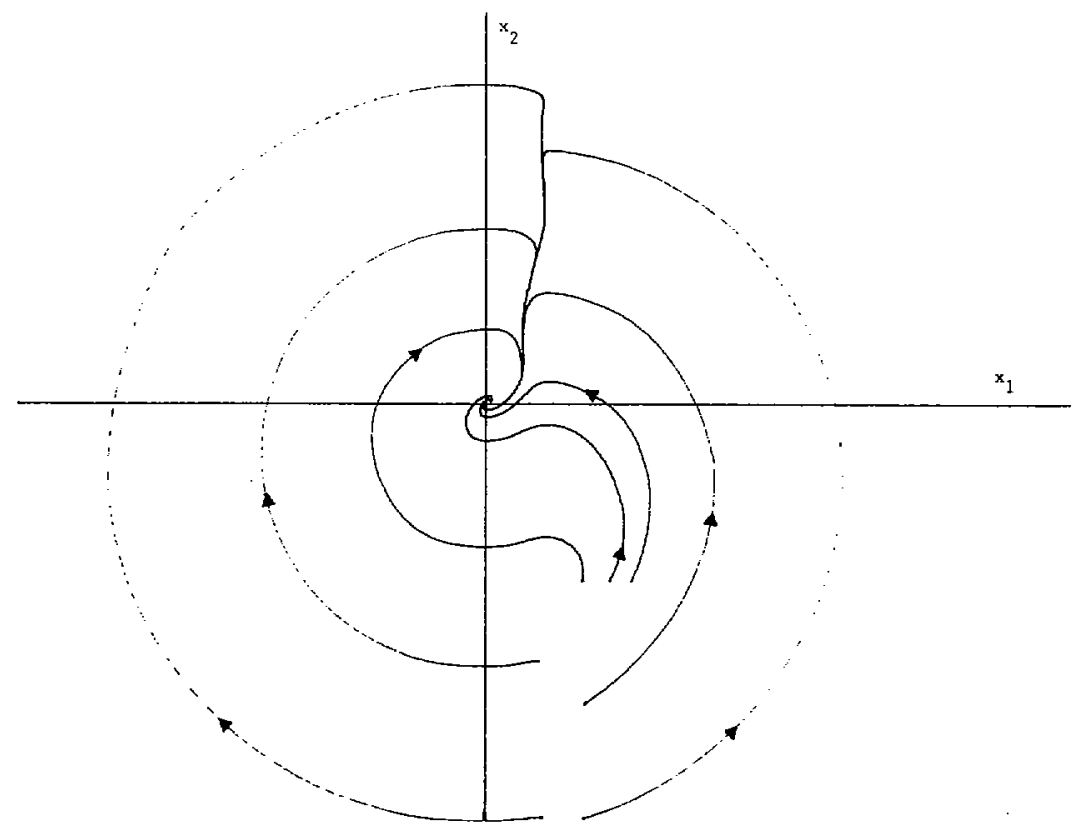

(d)

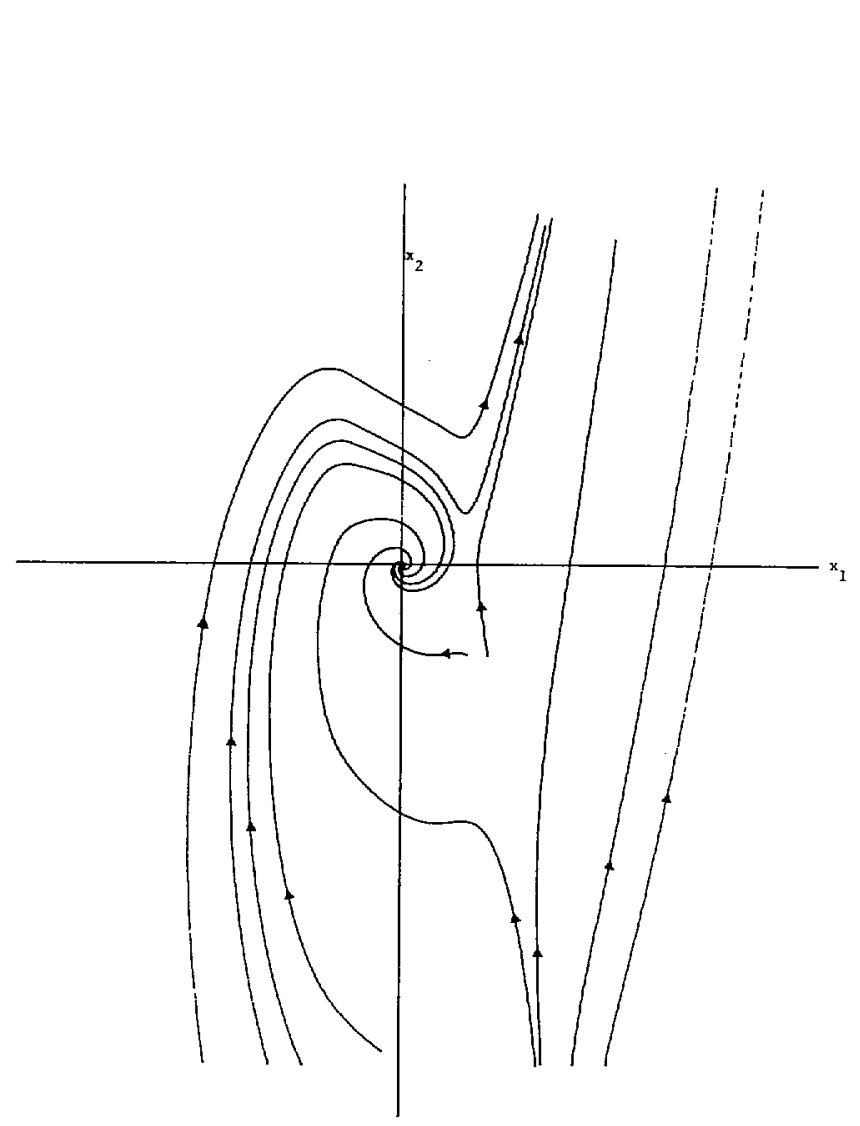

(e)

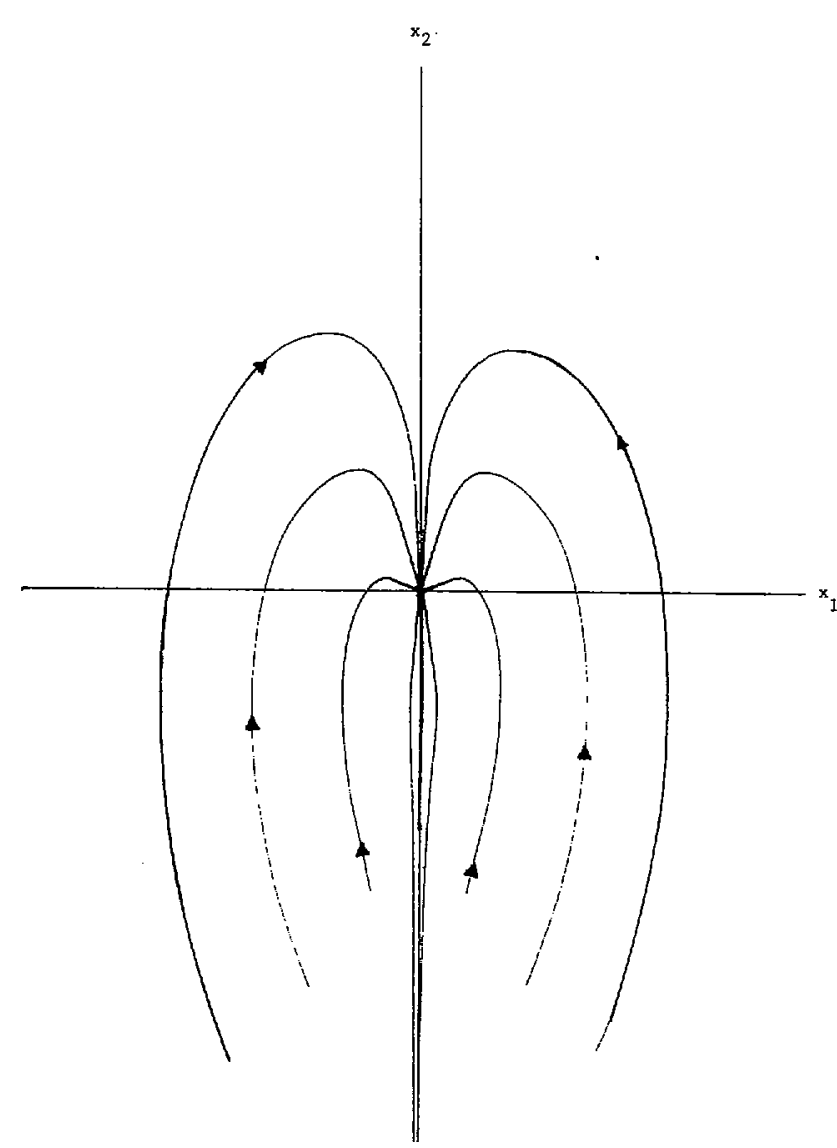

(f)

Fig. 5. (Continued.) (d) a.s.l. system with $x$-critical $D^{-1} A$ matrix (Example 4.3a). (e) System with unstable $A+\mu(x) D$ pencil, hence, with unbounded solutions (Example 4.4). (f) a.s.l. system with focal $D$ and $D^{-1} A$ matrices (Example 4.5a).

Lemma 4.2: If $|A|=0$ and $B(x)$ is type 1), then system (2) has either an unstable origin or off-origin equilibrium states.
Proof: Since $A$ is singular, let $A=a b^{T}$. Defining $V(x)$ $\triangleq a_{\perp}^{T} x$, we have $\dot{V}(x)=a_{\perp}^{T} A x+a_{\perp}^{T} B(x)=a_{\perp}^{T} B(x)=$ $x^{T} R x$ where $R=\left[a_{\perp 1} G+a_{\perp 2} H\right]_{s}$. If $R$ is positive or nega- 


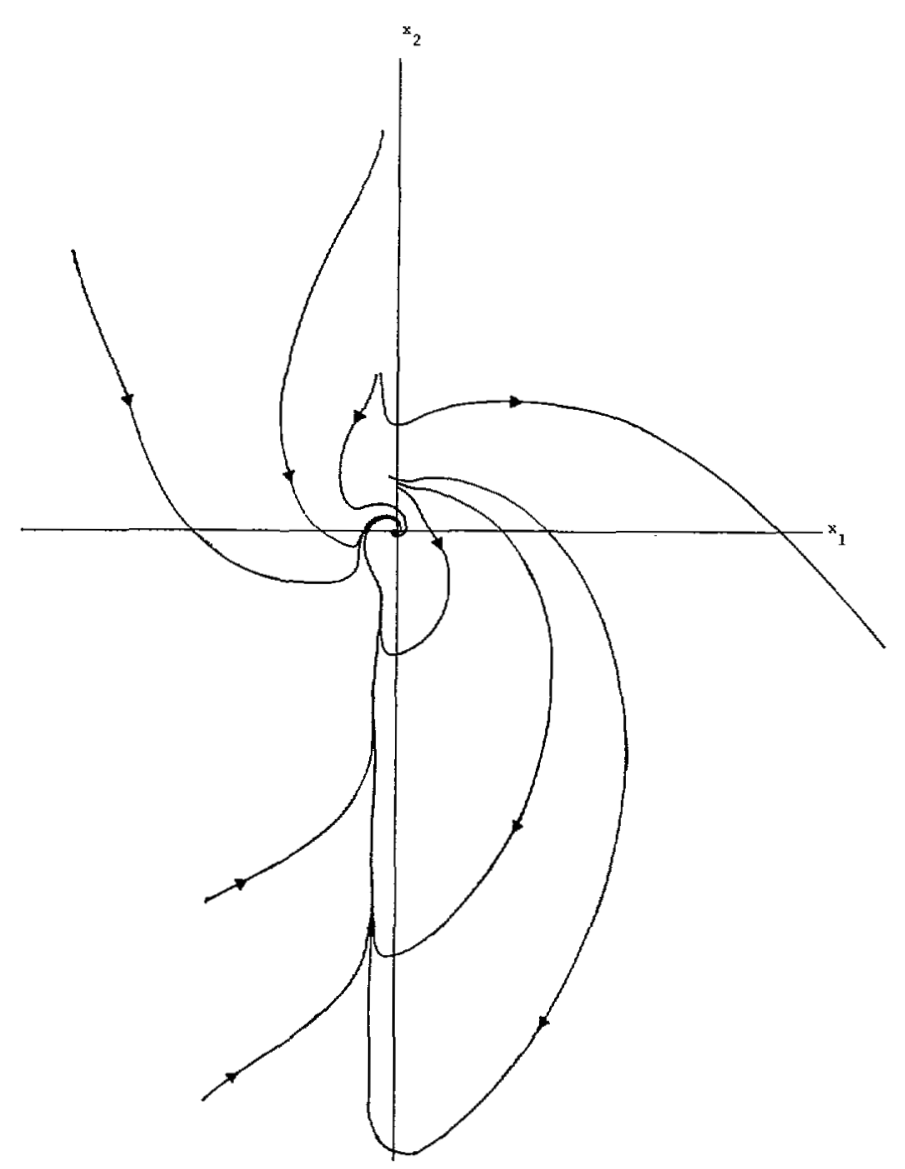

(g)

Fig. 5. (Continued.) (g) a.s.1. system with focal $A, D$, and $D^{-1} A$ matrices (Example 4.5b).

tive definite, then the system is unstable by Lyapunov's first instability theorem [4] since $V$ takes both positive and negative values in any neighborhood of the origin. If $R$ is positive or negative semidefinite, then the system is unstable by Krasovskii's extension of the first instability theorem [4] unless the line on which $\dot{V}$ vanishes contains a complete half-trajectory of (2). But $\dot{V} \equiv 0$ on $(x)$ implies $B(x) \in(a)$; hence if $(x)$ contains a complete half-trajectory of (2), then $f(x) \in(x)$ implies $(x)=(a)$ and that line must contain an off-origin equilibrium or an unbounded ray solution.

Finally, if $R$ is indefinite, then $B(x) \in(a)$ for two distinct lines in $\mathbb{R}^{2}$, one of which is not $\left(b_{\perp}\right)$. Then by Proposition 4.1 the system has an off-origin equilibrium state.

It should be noted that Lemmas 4.1 and 4.2 demonstrate the necessity of condition 2) in Theorem 3 . Since $B(x)$ cannot be type 1), we must have $B(x)=c^{T} x D x$ according to the results of Section III.

When $D=d b^{T}$ is singular, $B(x)=c^{T} x d b^{T} x=b^{T} x d c^{T} x$. In such a case there is an ambiguity in the parameterization of $B(x)$. If either $b$ or $c \in\left(d_{\perp}\right)$, then the corresponding $D$ matrix is critical and might allow a.s.l. behavior as in Example 4.2. To resolve this ambiguity, we shall classify a singular matrix $D$ as nodal only if both parameterizations are nodal and consider only the critical parameterization otherwise.
Lemma 4.3: Let $B(x)=c^{T} x D x$ and let $D$ be nodal. Then system (2) is not a.s.l.

Remark: This lemma is proved by considering the behavior of solutions near the dominant eigenvector of $D$. The case where $A$ has the same eigenvector almost always results in a ray solution on the corresponding line. In the more general case, the linear part of the field perturbs trajectories off this line. However, since solutions of the pure quadratic part tend toward the dominant eigenvector of $D$ (within a suitable neighborhood), a positive-invariant set may be constructed based on the cone formed by that line and a line perturbed slightly away from it in the direction of the linear part. The reader is referred to Appendix A for some of the facts about cones used in the proof of this case.

Proof: Since $c^{T} x D x=\left(-c^{T} x\right)(-D x)$, let $\sigma(D)$ $\triangleq\{\lambda, \mu\}, \lambda \geqslant|\mu|$, with no loss of generality, and suppose $D e=\lambda e$.

1) Case $A e \in(e)$ : If $e \notin\left(c_{\perp}\right)$, then $A e \neq 0$ implies the existence of an off-origin equilibrium by Proposition 4.1. If, however, $A e=0$, then since $e$ is an eigenvector of $D$, $(2)$ has a ray solution along $(e)$ and, hence, an unbounded solution.

If $e \in\left(c_{i}\right)$, then Proposition 4.1 does not apply. Using the change of basis $y \triangleq\left[c_{\perp}, c\right]^{-1} x$ we obtain

$\dot{y}_{2}=y_{2}\left(a_{22}+d_{22} y_{2}\right) \quad$ where $d_{22} \neq 0 \quad$ (since $D$ is nodal) 
and the system is unstable.

2) Case Ae $\notin(e)$ : Defining $V \triangleq 1 / 2 x^{T} P x-\gamma$ (where $\gamma>0$ ) yields $\dot{V}=x^{T} P A x+c^{T} x x^{T} P D x$. If $\Omega \triangleq \mathcal{C}_{\gamma}(P) \cap$ $\mathcal{K}_{+}(c)$, then $V>0$ on $\Omega$, and $V=0$ on $\partial \Omega$. Hence, if $P$ and $\gamma$ are chosen such that $\dot{V}>0$ on $\Omega$, the proof is complete according to Proposition 4.2.

To choose $P$, define $e_{\perp}$ such that $e_{\perp}^{T} A e>0$. Evidently, a small enough $\epsilon>0$ may be chosen such that if $b \triangleq e+\epsilon e_{\perp}$, then $c_{\perp} \notin \mathcal{C}_{0}(e, b)$. Moreover, if $P$ is chosen as $P \triangleq-\left[e_{\perp}\right.$ $\left.b_{\perp}^{T}\right]_{s}$, then $\mathcal{C}_{0}(e, b) \subseteq \bigodot_{0}\left([P D]_{s}\right)$, according to Fact A.2 (see Appendix A). ${ }^{2}$ Hence, the second term (cubic in $x$ ) of $\dot{V}$ is always nonnegative on $\Omega$. The following argument now demonstrates the existence of a large enough $\gamma>0$ such that this term dominates the first term-i.e., for all $x \in \Omega$, $c^{T} x x^{T} P D x>-x^{T} P A x$, or, equivalently, $c^{T} x>\theta(x)$ $\triangleq-x^{T} P A x / x^{T} P D x$. This argument is illustrated in Fig. 6 .

Since the denominator of $\theta$ is zero on $(e)$, and that line approaches $\partial \Omega$ arbitrarily closely, we will partition $\Omega$ using a line defined by some $g$ in its interior to be chosen below, as

$$
\Omega_{1} \triangleq \Omega \cap e_{0}(e, g) ; \quad \Omega_{2} \triangleq \Omega \cap e_{0}(g, b)
$$

and discuss $\Omega_{2}$, where $\theta$ is bounded separately. Note that $e^{T} P A e>0$, by construction of $P$, hence we may choose $g$ in $\Omega$, arbitrarily close to $(e)$, such that $x^{T} P A x>0$ for all $x \in \Omega_{1}$. It follows that $\dot{V}>0$ on $\Omega_{1}$ for any $\gamma>0$. Letting $\rho \triangleq \sup _{x \in \Omega_{2}} \theta(x)<\infty$, we must now choose $\gamma$ such that $c^{T} x>\rho$ on $\Omega_{2}$ which implies $\dot{V}>0$ on $\Omega_{2}$ as well. Since $c_{\perp} \notin \overline{\Omega_{2}}$, this is possible for any $\rho<\infty$.

Lemma 4.4: If $D$ is $x$-critical and nonsingular, then (2) is not a.s.1.

Proof: With no loss of generality, assume $D=\left[\begin{array}{ll}\delta & 1 \\ 0 & \delta\end{array}\right]$ so that $D$ is $\left[\begin{array}{l}1 \\ 0\end{array}\right]$-critical, and $\delta>0$. Let $A=\left[\begin{array}{ll}a_{11} & a_{12} \\ a_{21} & a_{22}\end{array}\right]$, and assume either $c_{1}>0$, or $c_{1}=0$ and $c_{2}>0 .{ }^{3}$ If $d \triangleq\left[\begin{array}{l}\delta \\ 1\end{array}\right]$ and $P \triangleq\left[d c^{r}\right]_{s}$, then the intersection of $\mathcal{C}_{0}(P)$ and the first quadrant is some nonempty cone. We will construct a positive-invariant set in this cone.

Let $\left(x_{0}\right)$ be in the interior of this cone, and define an affine ray, $\Lambda$, parallel to $\left(x_{0}\right)$, and a parabola, $\Gamma$, as

$$
\begin{aligned}
& \Lambda \triangleq\left\{\left[\begin{array}{c}
\alpha_{0} \\
0
\end{array}\right]+\alpha x_{0} \mid \alpha>0, \alpha_{0} \text { a positive constant }\right\} \\
& \Gamma \triangleq\left\{x \in \mathbb{R}^{2} \mid x_{1}=\beta x_{2}^{2}, x_{2}>0\right\} .
\end{aligned}
$$

These sets intersect at a unique point, $y$, determined by the magnitudes of $\alpha_{0}, \alpha$, and $\beta$, defining a region, $\Re$, disconnected from the origin, and bounded by $\Gamma_{+}, \Lambda_{+}$, and $\{y\}$

\footnotetext{
${ }^{2}$ The assumption that $D$ is nodal is crucial here. If $D$ is critical and nonsingular, then $\mathrm{e}_{0}(P) \subseteq \mathrm{e}_{\mathrm{D}}\left([P D]_{s}\right.$ only for one orientation of $e_{\perp}[\mathrm{e} . \mathrm{g}$., $e_{\perp} \triangleq J e$, rather than $e_{\perp} \triangleq-J e$ ) for which it may occur that $e_{\perp}^{T} A e<0$ (see Appendix A)]. Hence, the critical case is considered separately.

${ }^{3}$ Otherwise, if $c_{1}<0$, or $c_{1}=0$ and $c_{2}<0$, then an identical construction to the one given here may be completed in the third quadrant rather than the first.
}

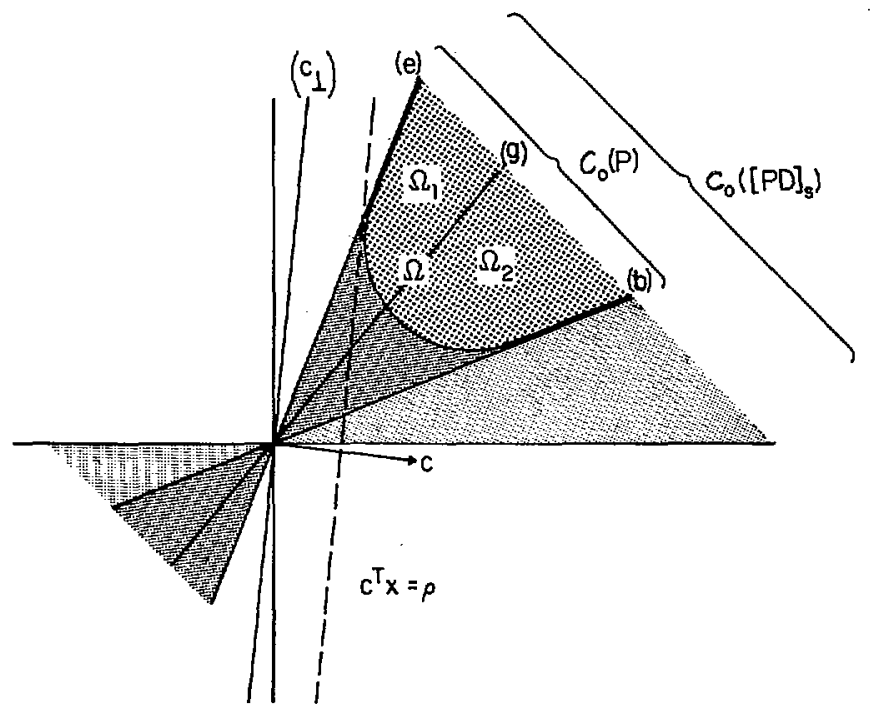

Fig. 6 .

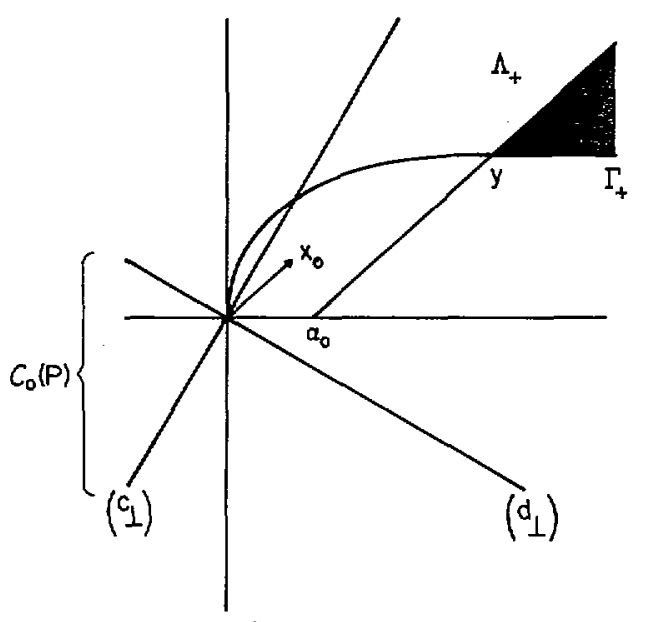

Fig. 7.

as shown in Fig. 7. We will show that $\dot{x}_{1}>0$ on $\Lambda_{+}$and $\dot{x}_{2}>0$ on $\Gamma_{+}$, completing the proof.

Considering the first inequality, if $x \in \Lambda_{+}$, we have $\dot{x}_{1}=f_{1}(x)=\delta c_{1} \alpha_{0}^{2}+\alpha^{2} x_{0}^{T} P x_{0}+\alpha_{0} \alpha\left(c_{1} d^{T} x_{0}+c^{T} x_{0} \delta\right)+$ $o\left(x_{0}, \alpha_{0}, \alpha\right)$; hence, $\dot{x}_{1}>0$ when $\alpha$ and $\alpha_{0}$ are suitably large. Evaluating the second component of the field on $\Gamma_{+}$, we have $\dot{x}_{2}=f_{2}(x)=\delta c_{1} \beta x_{2}^{3}+\left(a_{21} \beta+c_{2}\right) x_{2}^{2}+o(x)$. When $c_{1}>0$, it is clear that $\dot{x}_{2}>0$ on $\Gamma_{+}$if $y$ is suitably far from the origin-i.e., if $\alpha_{0}$ is large enough. If $c_{1}=0$, then $\beta<c_{2} / a_{21}$ implies $\dot{x}_{2}>0$ on $\Gamma_{+}$as well, since $c_{2}>0$ by assumption. Moreover, for small enough $\beta, y$ is far enough from the origin so that $\dot{x}_{1}>0$ on $\Lambda_{+}$as described above.

The preceding lemmas have excluded almost all examples of system (2) for which the quadratic part is unstable. It would be intuitively satisfying to add to the list of necessary conditions the requirement that the pure quadratic part of the field be stable. Unfortunately, as demonstrated by Example 4.2, a small class of unstable pure quadratic systems may, nevertheless, allow (2) to be a.s.l. At this point, the most we can require is that (2) admit the form 


$$
\dot{x}=A x+c^{T} x D x
$$

where $A$ is stable and $D$ is either focal or $x$-critical and singular. It is worth noting at this juncture that these results greatly restrict the class of bilinear systems (3) which are stabilizable under linear state feedback: namely, a bilinear system in $\mathbb{R}^{2}$ with a nodal matrix $D$ cannot be made a.s.l. by any choice $u=c^{T} x$, regardless of the properties of the pair $(A, b)$. In a subsequent paper, this question will be considered in greater detail [8].

We now proceed to prove the full statement of necessary conditions by considering the equilibrium states of (6). Since Proposition 4.1 establishes conditions for the existence of off-origin equilibria depending upon the zeros of $|A x, B(x)|$ we might expect $B(x)=c^{T} x D x$ to necessitate, in turn, $0 \neq|A x, D x|=|D|\left|D^{-1} A x, x\right|$ (when $|D| \neq 0$ ) i.e., that $D^{-1} A$ be focal. Yet, as demonstrated by Example 4.3 , in some cases $D^{-1} A$ may not be nodal, while (2) is still a.s.l. The following three lemmas will distinguish these special cases.

Lemma 4.5: If $D$ is focal, and $D^{-1} A$ is nodal, system (6) is not a.s.1.

Proof: Since $e \in \mathbb{R}^{2}$ is an eigenvector of $D^{-1} A$ iff $B(e) \in(A e)$, we have $B\left(e_{i}\right) \in\left(A e_{i}\right), i=1,2,\left(e_{1}\right) \neq\left(e_{2}\right)$ by assumption. According to Proposition 4.1, the system has an off-origin equilibrium unless simultaneously $A e_{1}=0$ and $e_{2} \in\left(c_{\perp}\right)$. In this case, $D^{-1} A=c_{\perp} b^{T}$ for some $b \in\left(e_{1 \perp}\right)$ and $A=D c_{\perp} b^{T}$.

Defining $V(x) \triangleq-x^{T}\left[D c_{\perp}\right]_{\perp} \quad$ implies $\quad \dot{V}(x)=$ $-c^{T} x\left[D c_{\perp}\right]_{\perp}^{T} D x=\left(c^{T} x\right) c^{T}\left[J^{T} D^{T} J D\right] x=|D|\left(c^{T} x\right)^{2}$ from Fact B.2 (see Appendix B). Thus $\dot{V} \geqslant 0$ (since $|D|>0$ for focal $D$ ) with equality only for $x \in\left(c_{\perp}\right)$. If $e_{2}$ is not an eigenvector of $A$, then a complete half-trajectory cannot remain on $\left(c_{\perp}\right)$, hence by Krasovskii's extension of Lyapunov's first instability theorem [4] the origin is unstable. If $A e_{2} \in\left(e_{2}\right)$, then $D=\alpha I$ and the field is purely quadratic on $\left(e_{1}\right)$ which must, therefore, contain a ray solution-the origin is unstable.

Lemma 4.6: If $D$ is focal and $D^{-1} A$ is $x$-critical, then (6) has no off-origin equilibrium states if and only if $x \in\left(c_{\perp}\right)$ when $|A| \neq 0$ and $x \notin\left(c_{\perp}\right)$ when $|A|=0$.

Proof: If $D^{-1} A$ is $x$-critical, then $B(y) \in(A y)$ if and only if $y \in(x)$. Under this condition, $|A|=0$ if and only if $A x=0$. Since $B(x)=0$ if and only if $x \in\left(c_{\perp}\right)$, we require $x \notin\left(c_{\perp}\right)$ when $A x=0$ and $x \in\left(c_{\perp}\right)$ when $A x \neq 0$ to ensure against an off-origin equilibrium state according to Proposition 4.1.

Lemma 4.7: If $D$ is singular and $x$-critical, then (6) is not a.s.l. unless $|A| \neq 0$ and $A^{-1} D=\gamma D$ for some scalar $\gamma$.

Proof: If $D$ is $x$-critical and singular, then for some $d \in(x)$ we have $D=d d_{\perp}^{T}$. If $A d \notin(d)$, then a proof identical to that of Lemma 4.3 may be given to show the existence of unbounded solutions. ${ }^{4}$ If $|A|=0$ and $A d \in(d)$, then either $A d=B(d)=0$, or $A x \in(d)=(B(x))$ for all $x \in \mathbb{R}^{2}$ - either case resulting in off-origin equilibria according to proposition 4.1. If $|A| \neq 0$ and $A d \in(d)$, then

\footnotetext{
${ }^{4}$ Note that this proof cannot necessarily be used for an $x$-critical matrix unless it is singular, as stated in the footnote to Lemma 4.3 , and shown in Appendix A.
}

$A^{-1} d \in(d)$; hence $A^{-1} D=\gamma d d_{\perp}^{T}=\gamma D$ for some scalar $\gamma$.

The reader should note that Lemmas $4.3-4.7$ prove the necessity of the conditions b). It remains to show that condition a) stated in Theorem 3 is necessary as well. This will conclude the proof of necessity of Theorem 3 .

Lemma 4.8: If $D$ is focal and $D^{-1} A$ is either nodal or $x$-critical, then system $(6)$ cannot be a.s.l. unless $[J D]_{s}>0$ implies $\left[D^{T} J A\right]_{s} \geqslant 0$ and $[J D]_{s}<0$ implies $\left[D^{T} J A\right]_{s} \leqslant 0$.

Proof: As shown in the proof of Corollary 4.1, if the stated condition does not hold, then $A+\mu(x) D$ has eigenvalues in $\overline{\mathbb{R}}^{+}$for all $x \in \mathbb{R}^{2}$. Hence, if $\mu_{0} \triangleq \mu\left(c_{\perp}\right)$, and $M_{0} \triangleq A+\mu_{0} D$, we have $\sigma\left(M_{0}\right)=\left\{\lambda_{0}, \delta_{0}\right\} \subseteq \overline{\mathbb{R}^{+}}$. Note that $\mu_{0} \neq 0$ since $A$ has negative eigenvalues. Since $M_{0} c_{\perp}=\lambda_{0} c_{\perp}$ from Proposition 4.3, it follows that $c^{T} M_{0}=\delta_{0} c^{T}$ according to Fact B.1 (see Appendix B). On the affine line $\left\{x \mid c^{T} x=\right.$ $\left.\mu_{0}\right\}$ we have $f(x)=M_{0} x=M_{0}\left(\mu_{0} c+\eta c_{\perp}\right), \eta \in \mathbb{R}$, and hence, $\mu_{0} c^{T} f(x)=\mu_{0}^{2} \delta_{0} \geqslant 0$. If $\delta_{0}>0$, then the half-plane separated from the origin is positive-invariant. If $\delta_{0}=0$, then the affine line is itself invariant. In either case, the system is not a.s.l.

\section{Sufficient Conditions for Asymptotic Stability in the Large}

This section is devoted to proving the sufficiency of the conditions in Theorem 3 . We will first prove that 1), 2), a), and b1) or b2) are sufficient in Proposition 4.4. We will show that the special case 1), 2), a), b3) is also a.s.l. in Proposition 4.5.

Proposition 4.4: The following conditions:

1) $A$ has eigenvalues in $\overline{\mathbb{C}^{-}}$;

2) $B(x)=c^{T} x D x$

3) $D$ is focal and $D^{-1} A$ is either focal or $x$-critical where $x \in\left(c_{\perp}\right)$ iff $|A| \neq 0$;

4) $A+\mu(x) D$ has eigenvalues in $\overline{\mathbb{R}^{-}}$; are sufficient for (2) to be a.s.l.

Remark: This result is obtained by constructing an arbitrarily large family of nested positive-invariant sets, each sharing one boundary point in common with the next. In the absence of off-origin equilibria, and since the shared boundary point precludes the possibility of a limit-cycle occurring between two consecutive sets, it follows that all trajectories enter each nested set in turn. Local attraction is demonstrated by showing that when this family cannot be made arbitrarily small, the boundary of the "smallest" set intersects an attractive domain generated by considering the stable linear part of the field. In the special case where the linear part is stable but not asymptotically stable, a separate lemma is required to demonstrate the absence of limit cycles locally. As discussed in the introduction of this section, condition 4) provides the crucial information that the linear field is always directed toward the interior of the logarithmic spiral generated by the focal system (5), $\dot{x}=D x$. Note that conditions 3 ) and 4) above are equivalent to a) and either b1) or b2) of Theorem 3, according to Proposition 4.3 and Corollary 4.1 .

Proof: Let $\sigma(D)=\{\alpha+i \beta, \alpha-i \beta\}$ where $\alpha \leqslant 0$ by proper choice of $c$ (as in Lemma 4.3) with no loss of 
generality. Choose an arbitrary vector, $x_{0} \in \mathbb{R}^{2}$, such that $c^{T} x_{0}>0$ and define the curve $\Delta_{n} \triangleq\left\{\bar{e}^{t D} x_{0} \mid t \in[n \beta / 2 \pi,(n\right.$ $+1) \beta / 2 \pi]\}$ with endpoints $\gamma^{n} x_{0}$ and $\gamma^{n+1} x_{0}$ where $1<\gamma$ [since $\alpha<0$ and $\beta / 2 \pi$ is the natural frequency of (5)] as shown in Fig. 8. ${ }^{5}$ Further define the line segment $\Lambda_{n}$ $\triangleq\left\{\zeta x_{0} \mid \gamma^{n+1} \leqslant \zeta \leqslant \gamma^{n}\right\}$ to close the curve and define the compact set $\mathscr{\Re}_{n}$ as shown in the figure. Since $\left\{\gamma^{n+1} x_{0}\right\}=$ $\Re_{n+1} \cap \Re_{n}$ and since Proposition 4.1 and Lemma 4.6 assure the absence of off-origin equilibria under the hypothesis above, if we demonstrate that each set is positiveinvariant it follows that $\Re_{0}$ is attractive.

The normal vector to the curve $\Delta_{n}$ at any point, $x$, lies in $\left([D x]_{\perp}\right)$ and, assuming $[J D]_{s}>0$, it follows that $n_{\Delta}(x)$ $\triangleq J^{r} D x$ is the normal to $\Delta_{n}$ at $x$ and directed toward the interior of $\Re_{n}$ since $x^{T} n_{\Delta}(x)=-x^{T} J D x<0$. ${ }^{6}$ Similarly, the interior directed normal on $\Lambda_{n}$ is given by $n_{\Lambda}(x) \triangleq J^{T} x_{0}$. To show that $\Re_{n}$ is positive-invariant it will suffice to show that $n_{\Delta}^{T}(x) f(x)$ and $n_{\Lambda}^{T}(x) f(x)$ are nonnegative for $x \in$ $\Delta_{n}, \Lambda_{n}$, respectively.

The first inequality holds for all $x \in \mathbb{R}^{2}$ since $n_{\Delta}^{T} f=$ $x^{T} D^{T} J f(x)=x^{T} D^{T} J A x$, and $x^{T} D^{T} J A x / x^{T} J D x=|A x, D x|$ $/|D x, x|=-\lambda(x)>0$ (according to Corollary 4.1, under these hypotheses) implies $x^{T} D^{T} J A x \geqslant 0$, given that $[J D]_{s}>$ 0 .

The second inequality holds on $\Lambda_{n}$ when $x_{0}$ is sufficiently large since $c^{T} x_{0}>0$ and $[J D]_{s}>0$ implies $n_{\Lambda}^{T}\left(x_{0}\right) f\left(x_{0}\right)=x_{0}^{T} J A x_{0}+c^{T} x_{0} x_{0}^{T} J D x_{0}>0$ when $c^{T} x_{0} \geqslant$ $-x_{0}^{T} J A x_{0} / x_{0}^{T} J D x_{0}=\mu\left(x_{0}\right)$. If $\mu\left(x_{0}\right) \leqslant 0$, then the inequality holds for arbitrarily small $x_{0}$, and the proof is complete.

If, however, for all $x_{0} \in \mathbb{R}^{2}, \mu\left(x_{0}\right)>0$, then $A$ must be focal and may be written as $A=\sigma I+\omega J$ in some coordinate system. Since $0>[J A]_{s}=-I \omega$ from the assumption $[J D]_{s}>0$ and $\mu>0$, it follows that $\omega>0$. If $\sigma<0$, then $V \triangleq 1 / 2 x^{T} x$ gives $\dot{V}=\sigma x^{T} x+c^{T} x x^{T} D x$, defining an open connected domain of attraction around the origin by $\Omega$ $\triangleq\{x \mid \dot{V}<0\}$. We shall show that for all $x_{0}$, any portion of $\Lambda$ on which the second inequality fails must be contained in $\Omega$. Suppose $n_{\Lambda}^{T}\left(x_{0}\right)^{T} f\left(x_{0}\right)<0$, i.e.,

$$
\begin{aligned}
0 & >x_{0}^{T} J A x_{0}+c^{T} x_{0} x_{0}^{T} J D x_{0} \\
& =-\omega x_{0}^{T} x_{0}+c^{T} x_{0} x_{0}^{T} J D x_{0} .
\end{aligned}
$$

Since

$$
0<x_{0}^{T} D^{T} J A x_{0}=\sigma x_{0}^{T} D^{T} J x_{0}-\omega x_{0}^{T} D x_{0}
$$

implies

$$
x_{0}^{T} D^{T} J x_{0}<\frac{\omega}{\sigma} x_{0}^{T} D x_{0},
$$

we have

$$
c^{T} x_{0} x_{0}^{T} J D x_{0}=c^{T} x_{0}\left(-x_{0}^{T} D^{T} J x_{0}\right)>\left(-\frac{\omega}{\sigma} x_{0}^{T} D x_{0}\right) c^{T} x_{0}
$$

\footnotetext{
${ }^{5}$ Note: if $\alpha=0$ then $\gamma=1$, and $D$ is skew-symmetric in some basis for which $V \triangleq x^{T} x$ is a global Lyapunov function-i.e., $\dot{V}=x^{T} A x<0$ under these hypotheses.

${ }^{6}$ If, instead, we assumed $[J D]_{s}<0$, then $J D x$ would specify the interior directed normal and the proof would proceed identically after interchanging $J$ for $J^{T}$.
}

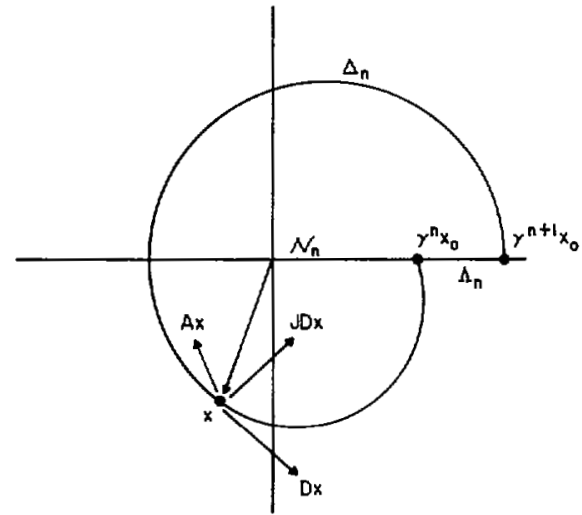

Fig. 8.

hence

$$
\begin{aligned}
0 & >-\omega x_{0}^{T} x_{0}+c^{T} x_{0}\left(x_{0}^{T} J D x_{0}\right) \\
& >-\frac{\omega}{\sigma}\left(\sigma x_{0}^{T} x_{0}+c^{T} x_{0} x_{0}^{T} D x_{0}\right)=-\frac{\omega}{\sigma} \dot{V}\left(x_{0}\right) .
\end{aligned}
$$

In the case $\sigma=0$, Lemma 4.9 demonstrates the absence of limit cycles, after which the result follows.

In order to complete the proof of Proposition 4.4, we rule out the possibility of periodic solutions, when the linear part of the field is critically stable, by the following lemma. This brief encounter is the closest brush with the phenomenon of quadratic limit cycles to be found in the paper. In a subsequent paper, we will use the same methods developed here to investigate this fascinating behavior more thoroughly and develop conditions for the existence of isolated periodic solutions of (2) when the origin is the unique equilibrium [7].

Lemma 4.9: Under the conditions of Proposition 4.4, if $A$ has pure imaginary eigenvalues, system (2) admits no periodic solutions.

Proof: Under some linear coordinate transformation, (2) may be rewritten as

$$
\dot{x}=J x+c^{r} x D x
$$

where condition 4) above implies $[J D]_{s}$ and $\left[D^{T} J^{2}\right]_{s}=-D_{s}$ are sign definite with the same sign (by Corollary 4.1). As before, we assume that $D$ is stable with no loss of generality and, hence, $D_{s}<0,[J D]_{s}>0$. Defining $V \triangleq 1 / 2 x^{T} x$ yields $\dot{V}=c^{T} x x^{T} D x$ which implies that $\|x\|$ grows in $\mathcal{H}_{-}$ $\triangleq\left\{x \mid c^{T} x<0\right\}$ and decreases in $\mathcal{K}_{+} \triangleq\left\{x \mid c^{T} x>0\right\}$. As shown in the proof of Proposition 4.4, $\Re_{0}$ is constructed such that $x_{0} \in \mathcal{O}_{0}$ implies $0>n_{\Lambda}\left(x_{0}\right)^{T} f\left(x_{0}\right)=\left|f\left(x_{0}\right), x_{0}\right|$. Thus every trajectory in $\Re_{0}$ crosses each line $\left(x_{0}\right)$ in the same direction (in this case, clockwise).

Choose a fixed point in $\mathcal{X}_{0}$ on $\left(c_{\perp}\right), x_{0} \triangleq \gamma_{0} J_{c}\left(\gamma_{0}>0\right)$. Since $\Re_{0}$ is positive-invariant, $p\left(t ; x_{0}\right) \in \Re_{0}$ for all $t>0$; hence $p\left(t ; x_{0}\right)$ must enter $\mathcal{K}_{-}$, and leave it at some time, $t_{1}>0$, on a point on the other side of $\left(c_{\perp}\right), x_{1} \triangleq-\gamma_{1} J c$ $\left(\gamma_{1}>0\right)$. Define the resulting curve $\Gamma_{-} \triangleq\left\{p\left(t ; x_{0}, \mid t \in\right.\right.$ $\left.\left(0, t_{1}\right)\right\} \subseteq \mathcal{H}_{-}$. We shall now join $x_{1}$ to $x_{0}$ by a curve, $\Gamma_{+}$in $\mathcal{H}_{+}$, on which the field is directed strictly toward the interior of the resulting neighborhood of the origin formed by the Jordan curve $g \triangleq \Gamma_{+} \cup \Gamma_{-}$. This will ensure that $p\left(t ; x_{1}\right) \cap \Gamma_{-}=0$, hence that $p\left(t ; x_{0}\right)$ is not periodic. 
Define a reflection of $\Gamma_{-}$into $\mathcal{K}_{+}$by the parameterization $r(t) \triangleq-\zeta(t) p\left(t ; x_{0}\right)$. Requiring $\zeta(0)=\gamma_{1} / \gamma_{0}=1 / \zeta\left(t_{1}\right)$ implies that $r(0)=x_{1}, r\left(t_{1}\right)=x_{0}$. Requiring that the ratio

$$
\xi \triangleq \frac{\|p-r\|}{\|p\| \cdot\|r\|}
$$

be constant specifies a family of smooth curves in $\mathcal{K}_{+}$of which a unique member satisfies the endpoint conditions above. Thus if $\Gamma_{+} \triangleq\left\{r(t) \mid r=-\zeta p ; \zeta(0)=1 / \zeta\left(t_{1}\right)=\right.$ $\left.\gamma_{1} / \gamma_{0} ; \dot{\xi} \equiv 0\right\}$, then $g$ is a Jordan curve defining a neighborhood containing the origin. This construction is illustrated in Fig. 9.

The tangent to $\Gamma_{+}$at any point, $r$, is given by $\dot{r}=-\dot{\zeta} p$ $-\zeta \dot{p}$. Thus for any $r \in \Gamma_{+}$we have

$$
r^{T} J \dot{r}=\zeta^{2} p^{T} J \dot{p}=-\zeta^{2}\left(p^{T} p-c^{T} p p^{T} J D p\right)<0
$$

since $[J D]_{s}>0$ and $p \in \mathscr{K}_{-}$, and $J \dot{r}$ is the interior directed normal vector for the set bounded by $g$ at $r$. It suffices to show that $f(r)^{T} J \dot{r}>0$ for all $r \in \Gamma_{+}$.

Since

$$
\begin{aligned}
\dot{r} & =r \dot{\xi} / \zeta-\zeta f(-r / \zeta) \\
& =r \dot{\zeta} / \zeta+J r \frac{1+\zeta}{\zeta}-f(r) 1 / \zeta,
\end{aligned}
$$

we have

$$
f(r)^{T} J \dot{r}=f^{T} J r \dot{\zeta} / \zeta-f^{T} r \frac{1+\zeta}{\zeta} .
$$

Since

$$
\begin{aligned}
0 & =\dot{\xi}=\frac{d}{d t} \frac{1+\zeta}{\|r\|} \\
& =\frac{1}{\|r\|}\left[\dot{\zeta}-\frac{1+\zeta}{r^{T} r} r^{T_{r}}\right] \\
& =\frac{1}{r}\left[-\dot{\zeta} / \zeta+\frac{1+\zeta}{\zeta} \frac{f^{T_{r}}}{r^{T} r}\right]
\end{aligned}
$$

we may substitute for $\dot{\zeta} \zeta$ in the first equality to get

$$
\begin{aligned}
f^{T} J \dot{r} & =\frac{f^{T} r}{r^{T} r} \frac{1+\zeta}{\zeta}\left(f^{T} J r-r^{T} r\right) \\
& =\frac{c^{T} r r^{T} D r}{r^{T} r} \frac{1+\zeta}{\zeta}\left(c^{T} r r^{T} D^{T} J r\right) \\
& =\frac{-\left(c^{T} r\right)^{2}(1+\zeta)}{\zeta r^{T} r}\left(r^{T} D r\right)\left(r^{T} J D r\right)>0
\end{aligned}
$$

since $D_{s}<0,[J D]_{s}>0$, and $r \in \mathcal{K}_{+}$.

We now conclude the proof of the sufficiency of the conditions of Theorem 3.

Proposition 4.6: If $D$ is critical and singular, then system (6) is a.s.l. if $|A| \neq 0$ and $A^{-1} D=\gamma D$ for some $\gamma \in \mathbb{R}$, in which case the pencil $A+\mu(x) D$ has eigenvalues in $\mathbb{R}^{-}$ when it is defined.

Proof: If $D=d d_{\perp}^{T}$ and $A^{-1} D=\gamma d d_{\perp}^{T}$, then $A d \in(d)$. Under the change of basis $y \triangleq\left[d, d_{1}\right]^{-1} x$, we have

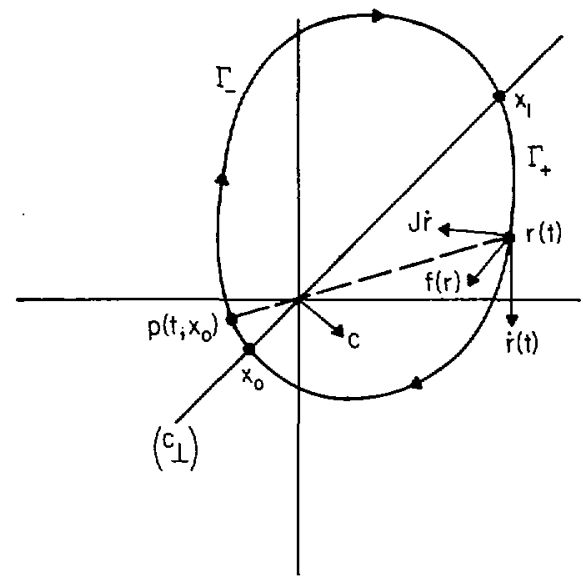

Fig. 9.

$$
\tilde{A}=\left[\begin{array}{cc}
a_{11} & a_{12} \\
0 & a_{22}
\end{array}\right], \quad \tilde{D}=\left[\begin{array}{cc}
0 & 1 \\
0 & 0
\end{array}\right]
$$

hence $\dot{y}_{2}=a_{22} y_{2}$ and $y_{2}(t)=e^{a_{22} t} y_{20} \rightarrow 0$. In this case,

$$
\dot{y}_{1}=\left(a_{11}+c_{1} e^{a_{22} t} y_{20}\right) y_{1}+a_{12} e^{a_{22} t} y_{20}+c_{2} e^{2 a_{22} t} y_{20}^{2}
$$

and $y_{1}(t) \rightarrow 0$. The system is a.s.l.

In the $y$ coordinate system, $\tilde{A}+\mu(y) \tilde{D}$ has the eigenvalue

$$
\lambda(y)=-\frac{|\tilde{A} y, \tilde{D} y|}{|\tilde{D} y, y|}=\frac{a_{22} y_{2}^{2}}{y_{2}^{2}}<0
$$

according to Proposition 4.3.

\section{Summary of Results}

Sections IV-A-C have investigated the properties and conditions for a.s.l. of system (2) according to the scheme depicted in Fig. 10 as stated in Theorem 3. To conclude our investigation we shall demonstrate that these conditions may be characterized more succinctly as stated in Theorem 2.

Theorem 2: The following conditions are necessary and sufficient for the asymptotic stability in the large of system (2):

1) the eigenvalues of $A$ have nonpositive real parts;

2) there exists a $c \in \mathbb{R}^{2}$ and $D \in \mathbb{R}^{2 \times 2}$ such that $B(x)$ $=c^{T} x D x$

3) the pencil $A+\mu(x) D$, where $\mu \triangleq-|A x, x| /$ $|D x, x|$, has nonpositive eigenvalues for all $x \in \mathbb{R}^{2}$ where it is defined; it is undefined, if ever, only on an eigenvector of $A$ in the null space of $D$ and $|A| \neq 0$; it is singular on, at most, a unique line, $\left(x_{0}\right)$, and $c_{\perp} \in\left(x_{0}\right)$ iff $|A| \neq 0$.

Proof-Necessity: We first show that conditions a) and b) of Theorem 3 are necessary for 3 ) above. Clearly, a) is necessary since, otherwise, $A+\mu(x) D$ will have positive eigenvalues, according to Proposition 4.3 and its corollary. Now assume that $b$ ) does not hold.

If $D$ is nodal with eigenvectors $d, e$, then $\mu(x)$ and hence the pencil is undefined on $(d)$ and $(e)$ violating 3 ) unless one or both linear factors of $|D x, x|$ are cancelled by factors of $|A x, x|$, i.e., unless $A$ shares one or both eigen- 


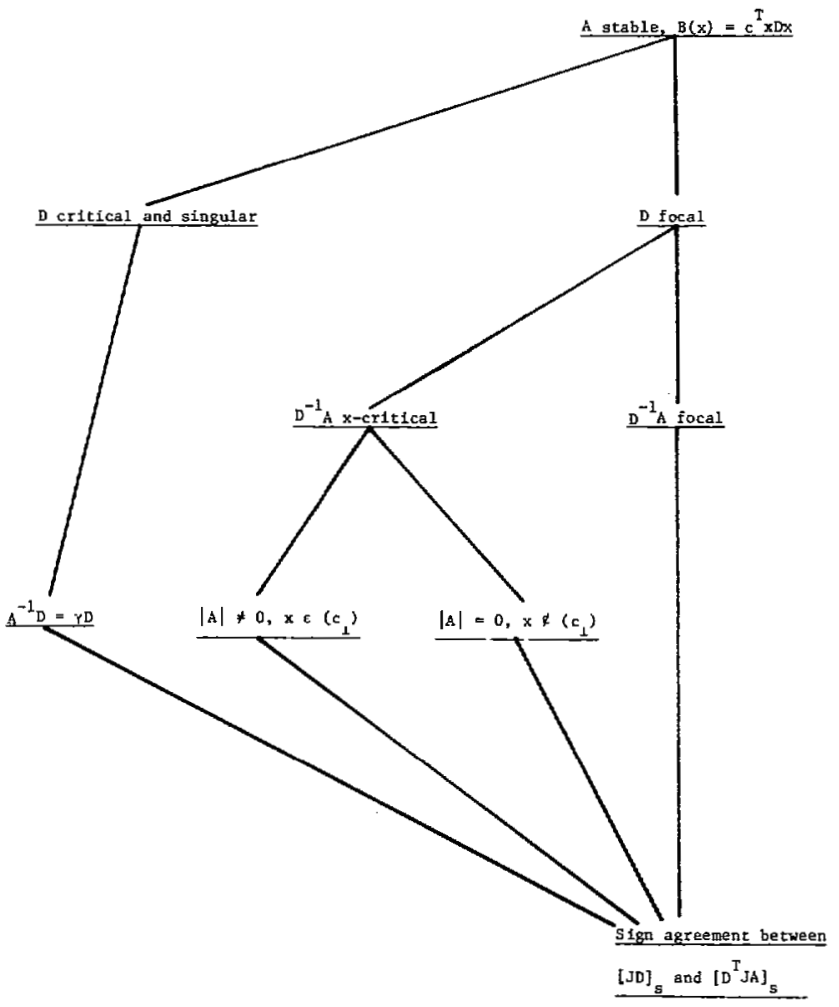

Fig. 10

vectors with $D$. In the latter case $A=\gamma D$; hence $\lambda(x)=0$ for all $x \in \mathbb{R}^{2}$, and the pencil is singular for all $x$. In the former case, if $A e=\alpha e$ but $A d \notin(d)$, then $\mu(x)$ is undefined on $(d)$. Both situations violate 3 ).

If $D$ is $x_{0}$-critical and nonsingular, then $|D x, x|=$ $\left(x^{T} x_{0 \perp}\right)^{2}$ and $\mu(x)$ must be undefined on $\left(x_{0}\right)$ unless $A=\gamma D$. Either possibility violates 3 ).

If $D$ is focal, then according to Proposition 4.3 , the pencil is singular iff $x$ is an eigenvector of $D^{-1} A$. Hence, $D^{-1} A$ cannot be nodal, and if it is $x_{0}$-critical we require $x_{0} \in\left(c_{\perp}\right)$ iff $|A| \neq 0$.

Sufficiency: We now show that a) and b) are sufficient for 3). If b1) holds, then $\mu$ is always defined, $A+\mu(x) D$ is never singular, and a) implies its eigenvalues are strictly negative. If b2) holds, then $\mu$ is always defined, $A+\mu(x) D$ is singular only on the unique eigenvector of $D^{-1} A$, and a) implies its eigenvalues are always nonpositive. If $\mathrm{b} 3$ ) holds, then $D=d d_{\perp}$ and $|D x, x|=\left(d_{\perp}^{T} x\right)^{2}$, and the pencil is undefined only on $(d)$, but $A d \in(d)$; hence, this is in agreement with 3). Finally in Proposition 4.6, it is shown that a) and b3) imply that $A+\mu(x) D$ has a constant, negative eigenvalue.

\section{APPENDIX A \\ SOME RESUlts CONCERNING CONES IN $\mathbb{R}^{2}$}

In Section IV-B the notion of a cone was introduced and used extensively. Unfortunately, the algebra and notation required to use this simple geometric construction in the body of the proofs in that section is unwieldy. This appendix presents the most important of the requisite results.

Definitions: Let $e, b$ be linearly independent in $\mathbb{R}^{2}$. Then

$$
\varrho_{\gamma}(e, b) \triangleq\{\alpha e+\beta b \mid \alpha \beta>\gamma \geqslant 0\} .
$$

$\bigodot_{0}(e, b)$ defines a conical region in $\mathbb{R}^{2}$. If $\gamma_{1}>\gamma_{2}, \bigodot_{\gamma_{1}}(e, b)$ is a proper subset of $e_{\gamma_{2}}(e, b)$. The distance of the set $e_{\gamma}(e, b)$ from the origin increases monotonically with $\gamma$ and tends to infinity as $\gamma$ tends to infinity.

An alternate description of $e_{0}(e, b)$, and $e_{\gamma}(e, b)$ uses $e_{\perp}$ and $b_{\perp}$.

$$
\begin{aligned}
& x \in \mathcal{C}_{0}(e, b) \text { iff }-x e_{\perp} b_{\perp}^{T} x>0 \\
& x \in \mathcal{C}_{\gamma}(e, b) \text { iff } \frac{-x e_{\perp} b_{\perp}^{T} x}{\left[e_{\perp}^{T} b_{\perp}\right]^{2}}>\gamma
\end{aligned}
$$

If $P \triangleq-\left[e_{\perp} b_{\perp}^{T}\right]_{s} /\left[e_{\perp}^{T} b_{\perp}\right]^{2}$, then $\bigodot_{\gamma}(e, b)=\bigodot_{\gamma}(P)$ defined in Section IV-A.

Fact A.1: Let $e, b$, and $x \in \mathbb{R}^{2}$. If $b$ and $x$ lie in the same half-plane defined by $(e)$ and $x \notin \bigodot_{0}(e, b)$, then

$$
e_{0}(e, b) \subseteq \bigodot_{0}(e, x) \text {. }
$$

Fact A.2: Let $D$ be critical or nodal with $\sigma(D)=\{\lambda, \mu\}$ and $\lambda \geqslant|\mu|$. Then given any $c \in \mathbb{R}^{2}$, there exists an indefinite matrix $P$ such that $c_{\perp} \notin \mathrm{C}_{0}(P)$ and $\mathrm{C}_{0}(P) \subseteq \mathrm{C}_{0}\left([P D]_{s}\right)$.

Comment: Fact A.2 is used in the proof of Lemma 4.3 to show that the given system is not a.s.1. The principal idea is to establish a region in $\mathbb{R}^{2}$ by the proper choice of $P$, which is positive-invariant. Embedding $\bigodot_{0}(P)$ in $\bigodot_{0}\left([P D]_{s}\right)$ establishes the positive invariance of one-half of the cone $C_{0}(P)$ under the influence of the pure quadratic field. Fact A.2 states that if $D$ is nodal or critical, such an embedding is always possible. This result could be used as an alternative proof of instability for the pure quadratic system when $D$ is not focal.

Proof: Let $D e=\lambda e$ and define $b \triangleq e+\epsilon e_{\perp}$ for some $\epsilon>0$. Note $b_{\perp}=e_{\perp}-\epsilon e$. Define $P \triangleq-\left[e_{\perp} b_{\perp}^{T}\right]_{s}$ to get

$$
P D=-\frac{1}{2}\left[e_{\perp} b_{\perp}^{T}+b_{\perp} e_{\perp}^{T}\right] D=-\frac{1}{2}\left[e_{\perp} b_{\perp}^{T} D+\mu b_{\perp} e_{\perp}^{T}\right]
$$

since $e_{\perp}^{T} D=\mu e_{\perp}^{T}$ from Fact B.1. Thus,

$$
[P D]_{s}=-\left[e_{\perp} g^{T}\right]_{s}
$$

where $g=\frac{1}{2}\left[D^{T}+\mu I\right] b_{\perp}$. If $g \in\left(e_{\perp}\right)$, then it directly follows that $0=e^{T} g=-\epsilon / 2(\lambda+\mu) e^{T_{T}^{T}} e$; hence $\lambda=-\mu$ and

$$
\bigodot_{0}\left([P D]_{s}\right)=\mathbb{R}^{2}-(e) \supseteq \bigodot_{0}(P) .
$$

If $g \notin\left(c_{\perp}\right)$, we consider the three cases where $D$ is 1$)$ critical, but not $x$-critical, 2) $x$-critical, and 3) nodal.

In case 1) $D=\alpha I(\alpha>0)$ and $P D=\alpha P$ so that $C_{0}(P)=$ $\mathcal{C}_{0}\left([P D]_{s}\right)$. For cases 2 ) and 3 ) we demonstrate that $\mathcal{C}_{0}(P)$ $\subseteq \mathcal{C}_{0}\left([P D]_{s}\right)$ by the equivalent demonstration that

$$
e_{0}(e, b) \subseteq \bigodot_{0}\left(e,-g_{\perp}\right)
$$

using A.1.

Since $e_{\perp}^{T} g_{\perp}=e^{T} g=-\epsilon / 2(\lambda+\mu)<0$ and $e_{\perp}^{T} b=\epsilon e_{\perp}^{T}$ $e_{\perp}>0$, it follows that $b$ and $-g_{\perp}$ lie in the same half-plane defined by $(e)$. To show $-g_{\perp} \notin \bigodot_{0}(e, b)$, it suffices to show $0>g_{\perp}^{T} P g_{\perp}=-\left(g_{\perp}^{T} e_{\perp}\right)\left(b_{\perp}^{T} g_{\perp}\right)$. Since $g_{\perp}^{T} e_{\perp}<0$ from above, this follows if and only if $b_{\perp}^{T} g_{\perp}=-\epsilon / 2 e^{T} e(\lambda$ 
$\left.-\mu+\epsilon e_{\perp}^{T} D e / e^{T} e\right)<0$. The last inequality is evidently true for small enough $\epsilon$ if $D$ is nodal since $\lambda>|\mu|$. If $D$ is $x$-critical, then $\lambda-\mu=0$ and the sign of $b_{\perp}^{T} g_{\perp}$ depends upon $J$-i.e., the orientation of $e_{\perp} \triangleq J e .^{7}$ In either case, $b_{\perp}^{T} g_{\perp}<0$ and $g_{\perp} \in e_{0}(e, b)$. Thus, by Fact A.1

$$
\bigodot_{0}(P)=\bigodot_{0}(e, b) \subseteq \bigcup_{0}\left(e,-g_{\perp}\right)=\bigodot_{0}\left([P D]_{s}\right) \text {. }
$$

To show that $c_{\perp} \notin e_{0}(P)$ we note that $c_{\perp}^{T} P c_{\perp}=-\left(c_{\perp}^{T}\right.$ $\left.e_{\perp}\right)^{2}+\epsilon\left(c_{\perp}^{T} e_{\perp} e^{T_{c_{1}}}\right) \leqslant 0$ for small enough $\epsilon$.

\section{APPENDIX B}

\section{SOME Properties of $(2 \times 2)$ Matrices}

Fact B.1: If $x$ is an eigenvector of the matrix $A, x_{\perp}$ is an eigenvector of the matrix $A^{T}$. If $\sigma(A)=\{\lambda, \mu\} \subseteq \mathbb{R}$ and $A x=\lambda x$, then $A^{T} x_{\perp}=\mu x_{\perp}$.

Proof: $\left(A x, x_{\perp}\right)=\left(\lambda x, x_{\perp}\right)=\left(x, A^{T} x_{\perp}\right)=0$ where $(x, y)$ is the inner product of the vectors $x$ and $y$. Hence, $x_{\perp}$ is an eigenvector of $A^{T}$.

Let the eigenvalue of $A^{T}$ corresponding to $x_{\perp}$ be $\alpha$ and let the eigenvector of $A$ corresponding to the eigenvalue $\mu$ be $y . A^{T} x_{\perp}=\alpha x_{\perp} ; A y=\mu y$.

$$
\left(A y, x_{\perp}\right)=\left(\mu y, x_{\perp}\right)=\left(y, A^{T} x_{\perp}\right)=\left(y, \alpha x_{\perp}\right) .
$$

Hence,

$$
(\mu-\alpha) y^{T} x_{\perp}=0 .
$$

Since the eigenvectors $y$ and $x$ are independent, $\mu=\alpha$.

Fact B.2: If $A$ is a nonsingular matrix and $J=\left[\begin{array}{rr}0 & -1 \\ 1 & 0\end{array}\right]$, then $A^{-1}=1 /|A| J^{T} A^{T} J$.

Proof: If

$$
\begin{aligned}
J & =\left[\begin{array}{rr}
0 & -1 \\
1 & 0
\end{array}\right], \\
A & =\left[\begin{array}{ll}
a_{11} & a_{12} \\
a_{21} & a_{22}
\end{array}\right] \\
J^{T} A^{T} J & =\left[\begin{array}{rr}
a_{22} & -a_{12} \\
-a_{21} & a_{11}
\end{array}\right] .
\end{aligned}
$$

\section{ACKNOWLEDGMENT}

The authors would like to express their gratitude to two of the reviewers for their careful attention and helpful suggestions.

\footnotetext{
${ }^{7}$ Unless $|D|=0$ in which case $D=e e_{\perp}^{T}$ and $e_{\perp}^{T} D^{T} e=\|e\|^{4}$ regardless of the choice of $J$.
}

\section{REFERENCES}

[1] W. A. Coppel. "A survey of quadratic systems," $J$. Differential Equations, vol. 2, pp. 293-304, 1966.

[2] T. Date, "Classification and analysis of two dimensional real homogeneous quadratic differential equation systems," $J$. Differential Equations, vol. 32, pp. 311-334, 1979.

[3] R. J. Dickson and L. M. Perko, "Bounded quadratic systems in the plane." J. Differential Equations, vol. 7, pp. 251-273, 1970.

[4] W. Hahn, Stability of Motion. New York: Springer-Verlag, 1967.

[5] J. K. Hale, Ordinary Differential Equations. New York: Wiley, 1969.

[6] D. E. Koditschek and K. S. Narendra. "The stability of second order quadratic differential equations," Dep. Eng. Appl. Sci., Yale Univ., New' Haven, CT, S \& IS Rep. 7709, Nov. 1977.

[7] - "Limit cycles of quadratic differential equations," Yale Univ., New Haven, CT, S \& IS Rep. 8110, in preparation.

[8] --. "Stabilizability of second order bilinear systems," Yale Univ., New Haven, CT, S \& IS Rep. 8109, in preparation.

[9] L. S. Lyaghina, "The integral curves of the equation $y^{\prime}=\left(a x^{2}+\right.$ $\left.b x y^{\prime}+c y^{2}\right) /\left(d x^{2}+e x y+f y^{2}\right)$ "' (in Russian), Uspekhi Mat. Nauk., vol. 6 , no. 2 , pp. $171-183,1951$.

[10] L. Markus, Quadratic Differential Equations and Non-Associative Algebras, Ann. Math. Studies, No. 45. Princeton, NJ: Princeton Univ. Press, 1960, pp. 185-213.

[11] I. G. Petrovskii and E. M. Landis, "On the number of limit cycles of the equation $d y / d x=P(x, y) / Q(x, y)$ where $P$ and $Q$ are polynomials of the second degree" (in Russian), Mat. $S b$., vol. 37, no. 79 , pp. 209-250, 1955; see also $A M S$ Transl. Ser. 2, vol. 10, pp. $177-221,1958$.

[12] N. I. Vulpe and K. S. Sibirskii, "Geometrical classification of quadratic differential systems" (in Russian), Diff. Urav., vol. 13, no. 5, pp. 803-814, 1977

[13] T. Chin-Chu, "The structure of the separatrix cycles of the system $d x / d t=\Sigma_{0 \leqslant i+k \leqslant 2} a_{i k} x^{i} y^{k}, d y / d t=\Sigma_{0 \leqslant i+k \leqslant 2} b_{i k} x^{i} y^{i}, "$ Chinese Math., vol. 3, pp. 277-284, 1963.

[14] Y. Yen-Chien, "A qualitative study of the integral curves of the differential equation $d y / d x=\left(q_{00}+q_{10} x+q_{01} y+q_{20} x^{2}+q_{11} x y\right.$ $\left.+q_{02} y_{2}^{2}\right) /\left(p_{00} p_{10} x p_{01} y+p_{20} x^{2}+p_{11} x y+p_{02} y^{2}\right), "$ Chinese Math, vol. 3 , pp. $1-18,1963$.

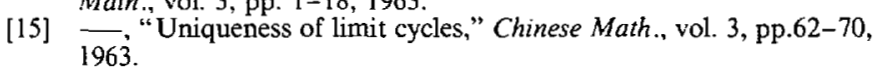

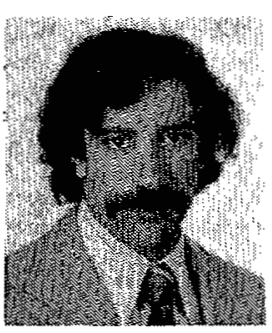

Daniel E. Koditschek received the B.S. degree in engineering and applied science from Yale University, New Haven, CT, in 1977.

He is currently pursuing doctoral research in mathematical systems theory at Yale, under the guidance of Prof. K. S. Narendra.

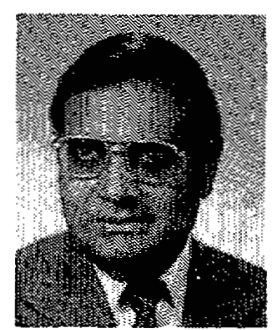

Kumpati S. Narendra (S'55-M'60-SM'63-F'79) received the $\mathrm{Ph} . \mathrm{D}$. degree in applied physics from Harvard University, Cambridge, MA, in 1959.

At present he is Professor of Electrical Engineering and Director of the Center for Systems Science at Yale University. New Haven, CT. 